NISTIR 8291

\title{
NIST 2019 Interlaboratory Study to Determine Starch Content in Corn Grain Biomass Intermediates
}

\author{
Jacolin A. Murray
}

This publication is available free of charge from: https://doi.org/10.6028/NIST.IR.8291
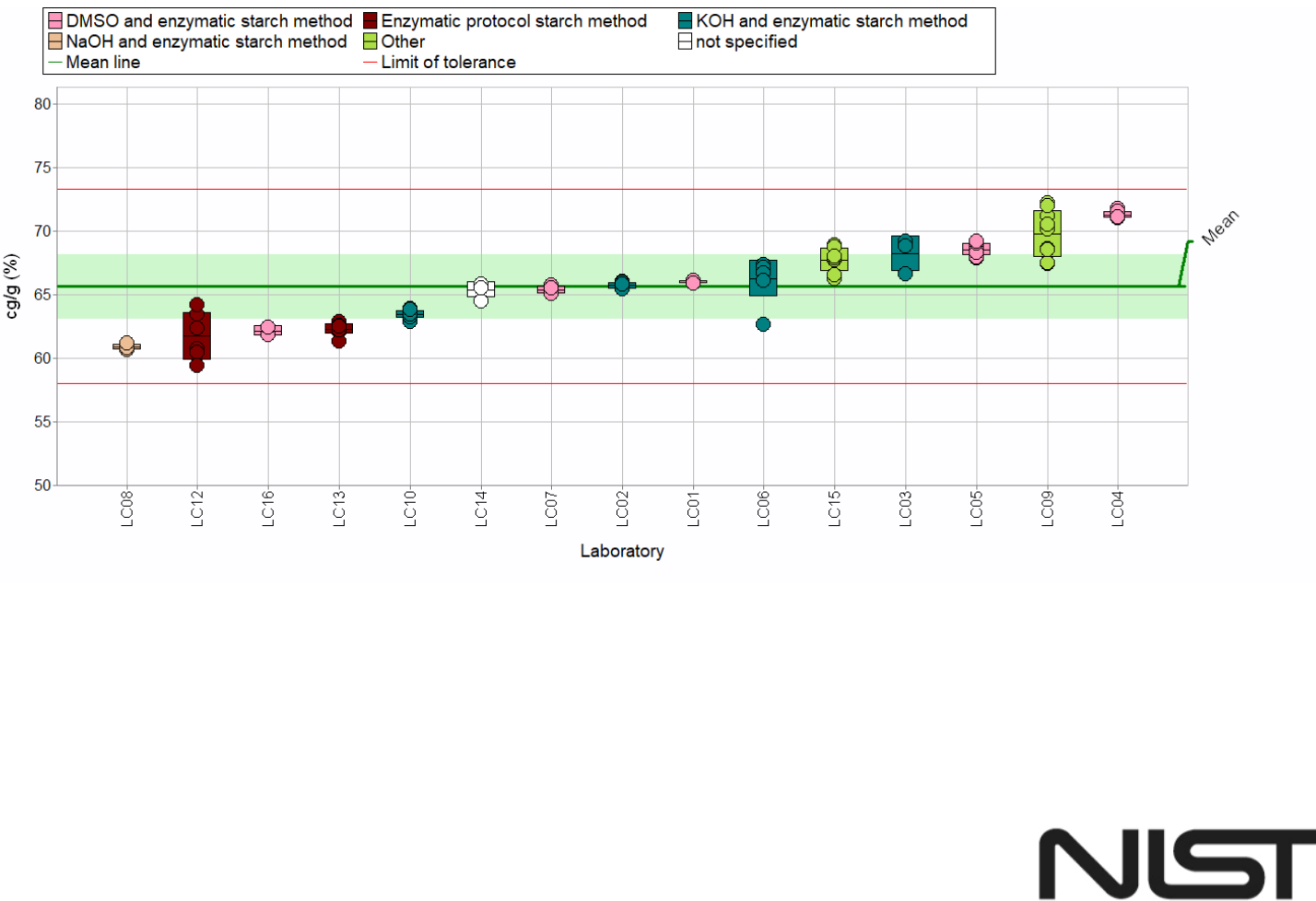

National Institute of Standards and Technology U.S. Department of Commerce 
This page intentionally blank. 


\title{
NIST 2019 Interlaboratory Study to Determine Starch Content in Corn Grain Biomass Intermediates
}

\author{
Jacolin A. Murray \\ Chemical Sciences Division \\ Material Measurement Laboratory
}

This publication is available free of charge from:

https://doi.org/10.6028/NIST.IR.8291

March 2020

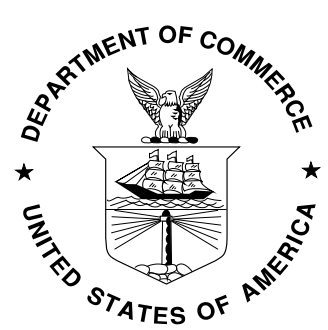

U.S. Department of Commerce Wilbur L. Ross, Jr., Secretary

National Institute of Standards and Technology Walter Copan, Under Secretary of Commerce for Standards and Technology and Director 
Certain commercial entities, equipment, or materials may be identified in this document in order to describe an experimental procedure or concept adequately. Such identification is not intended to imply recommendation or endorsement by the National Institute of Standards and Technology, nor is it intended to imply that the entities, materials, or equipment are necessarily the best available for the purpose.

National Institute of Standards and Technology Interagency or Internal Report 8291

Natl. Inst. Stand. Technol. Interag. Intern. Rep. 8291, 40 pages (March 2020)

This publication is available free of charge from: https://doi.org/10.6028/NIST.IR.8291 


\begin{abstract}
The National Institute of Standards and Technology (NIST) is currently developing reference materials (RMs) to support the corn-to-ethanol industry. Two candidate RMs consisting of dried corn biomass intermediates before and after conversion (fermentation) were obtained from commercial ethanol plant facilities. Interlaboratory studies will be used to screen the candidate RMs. This report describes the results of an interlaboratory study for laboratories analyzing starch content for these two candidate RMs and for two starch-based control materials.
\end{abstract}

\title{
Keywords
}

Starch, Cellulose, Cellulosic Content, Biomass, Ethanol production, Interlaboratory Study 


\section{Table of Contents}

Introduction .............................................................................................................................................. 1

Source of Materials ............................................................................................................................. 2

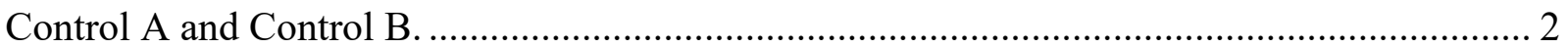

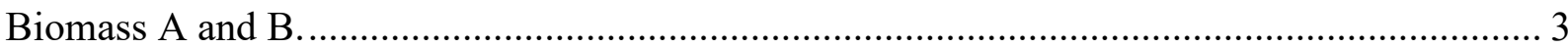

Instructions to Participants............................................................................................................. 4

Overview of Data Treatment and Representation......................................................................... 6

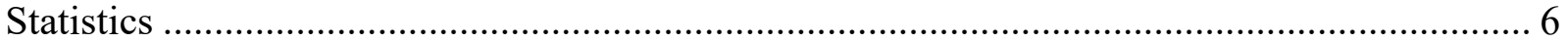

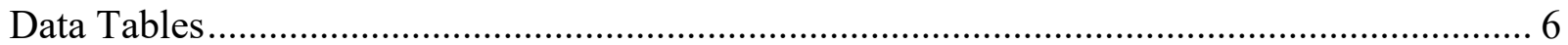

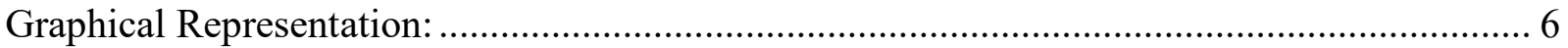

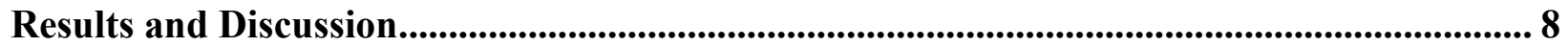

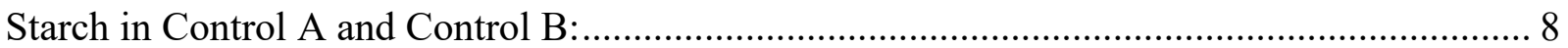

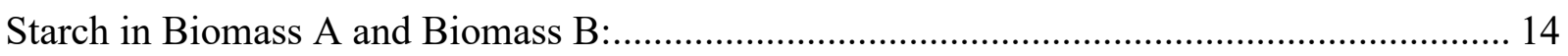

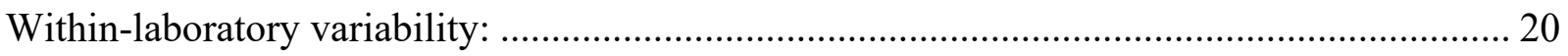

Within-and Between-Sample Variability for Starch Analysis: ................................................ 20

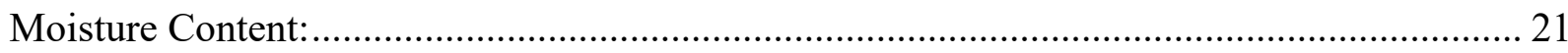

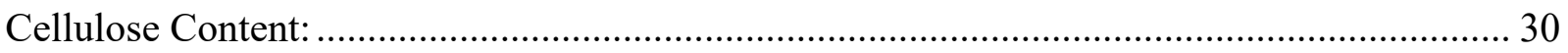

Conclusions......................................................................................................................................... 31

Acknowledgements ................................................................................................................................ 33

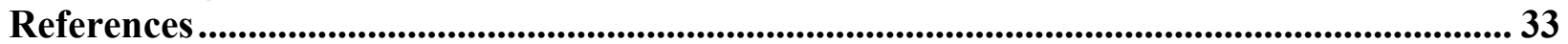

\section{List of Tables}

Table 1: Mass fraction of starch for Control A and Control B. .............................................................

Table 2: Data Summary for mass fraction of starch in Control A. .......................................................... 8

Table 3: Data Summary for mass fraction of starch in Control B reported on an as-is basis. .................... 11

Table 4: Data Summary for mass fraction of starch in Biomass A....................................................... 15

Table 5: Data Summary for the mass fraction of starch in Biomass B. ................................................. 17

Table 6: RSD for all replicates reported by each lab for each material...................................................2

Table 7: Data summary for the mass fraction of moisture in Control A...................................................2

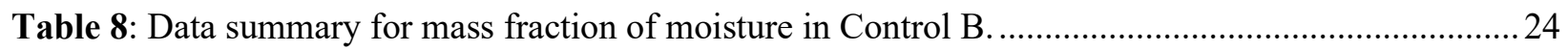

Table 9: Data summary for mass fraction of moisture in Biomass A. .......................................................26

Table 10: Data summary for mass fraction of moisture in Biomass B. ................................................... 28

Table 11: Consensus mean, SD, and RSD of starch reported as-is and on a dry-mass basis ......................30 


\section{List of Figures}

Figure 1: Pie charts representing the number of participants (total $\mathrm{N}=15$ ) using each method............... 5

Figure 2: Mass fraction of starch in Control A reported on an as-is basis............................................. 9

Figure 3: Mass fraction of starch in Control B reported on an as-is basis............................................ 12

Figure 4: Laboratory means for mass fraction of starch in Control A and in Control B....................... 13

Figure 5: Mass fraction of starch in Biomass A reported on an as-is basis. ......................................... 16

Figure 6: Mass fraction of starch in Biomass B reported on an as-is basis. .......................................... 18

Figure 7: Laboratory means of the mass fraction of starch in Biomass A and in Biomass B.................. 19

Figure 8: Between-Sample Variability as a function of Within-Sample Variability ..............................2 21

Figure 9: Mass fraction of moisture content in Control A.................................................................2 23

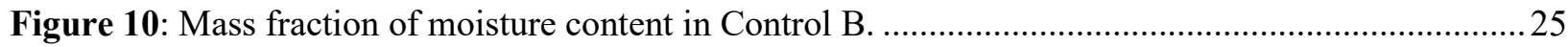

Figure 11: Mass fraction of moisture content in Biomass A..............................................................2 27

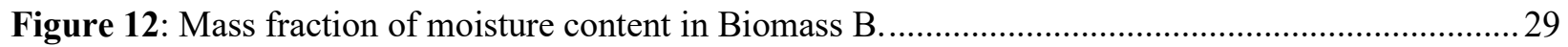

\begin{tabular}{ll} 
ANOVA & \multicolumn{1}{c}{ Abbreviations, Acronyms, and Symbols } \\
AOAC & AOAlysis of Variance \\
Avg & Average \\
cg/g & Centigrams per gram (\%) \\
D3 fuel & Cellulosic biofuel as defined by the RFS program \\
D6 fuel & Non-advanced renewable fuel as defined by the RFS program \\
DMSO & Dimethyl sulfoxide \\
EPA & United States Environmental Protection Agency \\
ISO & International Organization for Standardization \\
KOH & Potassium hydroxide \\
Lab & Participant code \\
LC & liquid chromatography \\
$N$ & Number of participants or replicates \\
NaOH & sodium hydroxide \\
NFTA & National Forage Testing Association \\
NIST & National Institute of Standards and Technology \\
No. & Number \\
NREL & National Renewable Energy Laboratory \\
Rep. & Replicate \\
RFS & Renewable Fuel Standard \\
RIN & Renewable identification number \\
RM & Reference material \\
RSD & Percent relative standard deviation \\
$s *$ & Consensus standard deviation \\
SD & Standard deviation \\
$U$ mean & Expanded uncertainty of consensus mean \\
$u$ mean & Standard uncertainty of consensus mean \\
$Z$ & Z-score \\
\hline
\end{tabular}




\section{Introduction}

The National Institute of Standards and Technology (NIST) is developing reference materials (RMs) to support cellulosic ethanol production in response to updates to the Renewable Fuel Standard (RFS) program ${ }^{1}$ that were enacted in $2014 .^{2}$ The RFS program, originally introduced in 2005 , is designed to reduce greenhouse gas emissions and decrease the amount of petroleum fuel the United States imports by requiring a specified volume of renewable fuels to be used in transportation fuel sold in the in United States each year. ${ }^{1}$ There are four different categories of fuels available under the RFS program; the categories are differentiated based on the fuel type generated, feedstock used, energy inputs, and the overall greenhouse gas reduction. ${ }^{1}$ The amount of renewable fuel that is generated and used to demonstrate compliance to this national policy is documented through credits called Renewable Identification Numbers (RINs). ${ }^{1}$ In 2014, corn kernel fiber became an approved cellulosic feedstock. ${ }^{3}$ This allows domestic ethanol production facilities that produce ethanol from corn kernel feedstock the opportunity to earn both D6 RINs (credits earned by producing renewable fuel, i.e. corn ethanol) and D3 RINs (credits earned for producing cellulosic biofuel), if the facility can produce ethanol from both the starch and cellulosic components, respectively. Producers are then required to calculate converted fraction of fuel generated from each component of the feedstock to ensure that the correct RINs are generated. Hence, it is crucial that the analytical protocols used to measure the starch and cellulosic content in samples collected from ethanol production facilities are accurate. The availability of matrixrelevant RMs are important analytical tools to validate methods and/or confirm results and would be beneficial to the bioethanol community. The United States Environmental Protection Agency (EPA) has released further guidance on qualifying analytical methods for determining cellulosic converted fraction of corn kernel fiber co-processed with starch. ${ }^{4}$

The EPA and the National Renewable Energy Laboratory (NREL) have requested that NIST develop RMs to support the simultaneous conversion of starch and cellulosic content to ethanol. NIST has worked closely with both government and industry stakeholders in designing and developing fit-for-purpose RMs. The source materials for two candidate RMs, consisting of corn grain biomass intermediates before and after conversion (fermentation), were obtained from commercial ethanol production facilities. The materials were dried, ground, and blended prior to packaging. Material characterization is occurring in two phases, the first phase is starch determination, followed by cellulosic content determination. Interlaboratory studies will be used to screen the candidate RMs.

The results of an interlaboratory study for starch analysis of the candidate RMs are summarized in this report. The primary goals of the interlaboratory study are: 1) to support measurement comparability of starch in renewable fuel intermediates and 2) to determine a consensus starch content for the candidate RMs. For this study, participants were asked to measure starch on an asis basis in the two candidate materials and in two control materials. Moisture content was optional as well as cellulosic content. Fifteen participants reported values for starch. Samples were shipped to participants in March 2019 and results were returned to NIST in April 2019. 


\section{Source of Materials}

Study samples were provided to the participants free of charge. Participants received a total of twelve samples: two packets of Control A (labeled as Control A-Samples 1-2), two packets of Control B (labeled as Control B-Samples 1-2), four packets of Biomass A (labeled as Biomass ASamples 1-4), and four packets of Biomass B (labeled as Biomass B-Samples 1-4). The Control materials were designed to enable evaluation of participant performance; study participants were not informed of the starch content in any of the study materials.

Participants were instructed to store these materials at room temperature and to mix the contents of the packet with a spatula before removing test portions for analysis. A sample size of at least $0.1 \mathrm{~g}$ for analysis was recommended.

Because one of the controls contains resistant starch and both biomass materials are suspected to contain some resistant starch, participants were asked to use analytical protocols amendable to resistant starch. Resistant starch is defined in this report as starch that cannot be readily broken down to glucose by commercially available enzymes such as alpha amylase and amyloglucosidase. Examples of methods that are amendable to resistant starch include pretreatment of samples using dimethyl sulfoxide (DMSO) and sodium or potassium hydroxide $(\mathrm{NaOH}$, and $\mathrm{KOH})$.

\section{Control $A$ and Control $B$.}

Control A and Control B are gravimetrically prepared blends of commercially available starch and cellulose. Control A consists of two sources of starch, a soluble starch and a resistant corn starch. It is estimated that the mass fraction of resistant starch in Control A is approximately 13 centigrams per gram $(\mathrm{cg} / \mathrm{g})$, which is equivalent to "\%". This was determined from analyzing the resistant corn starch source material with and without dissolving the resistant starch with dimethyl sulfoxide (DMSO). It was assumed that the analysis using DMSO accessed both soluble and resistant starch and the analysis without using DMSO only accesses the soluble starch. The analytical method used to determine starch content was a modified version of the AOAC INTERNATIONAL (AOAC) 996.11 protocol. $^{5}$ Control B only contained soluble starch.

For both control materials, approximately $900 \mathrm{~g}$ of material were prepared in 2 L Nalgene bottles. After the starch and cellulose were added, the bottles were mixed aggressively on a large capacity mixer for $2 \mathrm{~h}$ before aliquoting into Mylar packets. Each Mylar packet consisted of approximately $10 \mathrm{~g}$ of material. Table 1 list the gravimetric starch mass fractions in Control A and Control B.

Table 1: Mass fraction of starch for Control A and Control B.

\begin{tabular}{c|c|c|}
\multicolumn{1}{c}{ Starch, cg/g $^{(\mathrm{a})}$} \\
\cline { 2 - 3 } & Control A $^{(\mathrm{b})}$ & Control B \\
\hline Total Starch & $67.6 \pm 3.6$ & $8.98 \pm 0.34$ \\
\cline { 2 - 3 }
\end{tabular}

a) Values are expressed as $x \pm U_{95 \%}(x)$, where $x$ is the mean gravimetric value and $U_{95 \%}(x)$ is the expanded uncertainty of the value. The true value of the analyte is believed to lie within the interval $x \pm U_{95 \%}(x)$ with $95 \%$ confidence.

b) Contains approximately $13 \mathrm{cg} / \mathrm{g}$ resistant $\mathrm{starch}$ 
It was assumed that the only significant source of impurities of the starting materials was moisture. The moisture content of the starting materials was determined by heating the samples in a forcedair oven at $105^{\circ} \mathrm{C}$ for $3 \mathrm{~h}$, similar to methods outlined in AOAC 935.29 ${ }^{6}$ and the National Forage Testing Association (NFTA) Reference Method 2.2.2.5. ${ }^{7}$ The gravimetric mass fractions of starch in Control A and Control B were determined by dividing the mass of the starch, with purity correction for moisture, by the total mass of the mixture. For convenience, this mass fraction is expressed in units of $\mathrm{cg} / \mathrm{g}(\%)$. This represents the mass fraction of starch "as-is" in the materials.

The homogeneity of Control A and Control B was accessed by analyzing select packets across the entire production lot using a sampling size of $0.1 \mathrm{~g}$. Using one-factor Analysis of Variance (ANOVA), these data were used to estimate within- and between-packet standard deviations (SDs), which were used to assign the expanded uncertainties listed in Table $1{ }^{8}$ The uncertainties with weighing the starch and the total mixture and moisture content analysis were negligible.

The mass fraction of cellulose added to Control A and Control B was determined to be $22.8 \mathrm{cg} / \mathrm{g}$ and $86.1 \mathrm{cg} / \mathrm{g}$, respectively. No cellulose analyses were performed on the materials, hence the uncertainties in these values were not estimated.

\section{Biomass $A$ and $B$.}

The source materials for Biomass A and Biomass B were donated to NIST. Biomass A is a dried corn grain biomass intermediate obtained from a commercial ethanol production facility prior to conversion, or fermentation. Biomass B is a dried corn grain biomass intermediate obtained from a commercial ethanol production facility after conversion, or fermentation. The source materials were collected in July 2018 and dried in a vacuum double-drum dryer before being ground using a centrifugal mill with a $0.75 \mathrm{~mm}$ screen size. During drying, the materials were briefly exposed $(\approx 5 \mathrm{~s})$ to the dryer rolls that were at $\approx 112{ }^{\circ} \mathrm{C}$ that may have caused the formation of some resistant starch in the materials. The materials were then homogenized in a rotary tumble-drum homogenizer for $30 \mathrm{~min}$. The dried corn grain biomass intermediates were shipped to NIST. The materials were blended once again at NIST prior to packaging in Mylar bags in $10 \mathrm{~g}$ aliquots. Biomass A was packaged in November 2018 and Biomass was packaged in October 2018. The packaged Biomass A and Biomass B were irradiated for microbiological control December 7-8, 2018 with a minimum and maximum absorbed dose measured of $10.9 \mathrm{kGy}$ and $13.0 \mathrm{kGy}$, respectively. Biomass $\mathrm{A}$ and Biomass $\mathrm{B}$ were stored at room temperature after packaging and irradiation. 


\section{Instructions to Participants}

The focus of this interlaboratory study was measuring total starch in both control and biomass samples. While a total of twelve samples were sent to participants, the participants were requested to make a single measurement and report a total starch value for eight of the samples: Biomass ASample 1, Biomass A-Sample 2, Biomass A-Sample 3, Biomass B-Sample 1, Biomass B-Sample 2, Biomass B-Sample 3, Control A-Sample 1, and Control B-Sample 1. If time and resources permitted, participants could report replicates for each sample, but it was not required.

The participants were requested to report starch on an as-is basis. The determination of moisture was not required, but participants could report results if time and resources permitted. Reporting cellulose or cellulosic content (including cellulose, hemicellulose, and lignin) of the interlaboratory samples was optional and not required. An additional sample of each material (Biomass A-Sample 4, Biomass B-Sample 4, Control A-sample 2, and Control B-Sample 2) was provided for participants that required extra material to perform moisture or cellulosic content analyses. For the controls, participants were instructed to analyze a single aliquot of Control ASample 1 and a single aliquot of Control B-Sample 1 for total starch. Reporting replicates of each sample was optional.

NIST requested participants to measure total starch to include mono- and oligosaccharides by their current protocols. Some starch methods include a washing step to remove the soluble saccharides prior to starch analysis. Since C6 glucan mono- and oligosaccharides are included in the starch total, NIST requested that a washing step not be used for these measurements. The analytical methods used should be amendable to resistant starch, e.g., the use of DMSO or KOH. Total starch values were reported as a percent by mass on an as-is basis; the participants were asked not to correct for moisture. Participants that performed replicates of the same packet (for example, performing three replicates on Biomass A-Sample 1) were asked not to report an average but report each replicate separately in the space provided in the reporting sheet.

Moisture analysis was optional. It was suggested that participants who wished to provide moisture information use AOAC 935.296 or NFTA 2.2.2.57 protocols. Determining cellulose or cellulosic content was also optional. Cellulosic content consists of cellulose, hemicellulose, and lignin. Participants were asked to use their current protocols for cellulose or cellulosic content and report results on an as-is basis.

Participants reported results on a web-based platform, RingDat (Quadrata GmbH, Dresden, Germany). On the reporting sheet, participants could report values for starch, moisture content, and cellulosic content for each sample. Results were reported as mass-fractions expressed as percentages $(\mathrm{cg} / \mathrm{g})$. In addition, the date of analysis, and method used (from a choice from a dropdown menu) were additional user inputs. Figure 1 lists the methods and the number of participants that selected each method for the sample preparation and detection methods. 
A

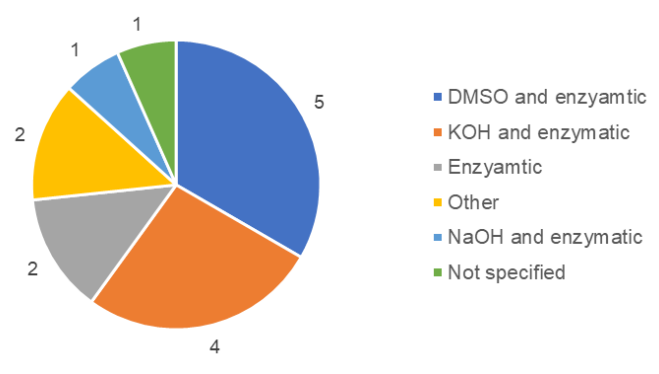

B

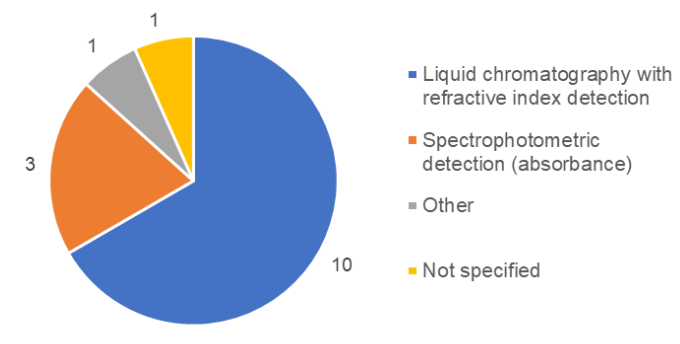

Figure 1: Pie charts representing the number of participants (total $N=15$ ) using each method. A) Method descriptions for the sample preparation method for the analysis of total starch that participants could choose from in the data submission form and the number of participants using each method. B) Method description for the detection method for the analysis of total starch that participants could choose from in the data submission form and the number of participants choosing each method. 


\section{Overview of Data Treatment and Representation}

Data tables and graphs are provided throughout this report using anonymized laboratory codes.

\section{Statistics}

Data tables and graphs throughout this report contain the results of the interlaboratory study. All calculations were performed in PROLab Plus (QuoData GmbH, Dresden, Germany). The consensus mean and SD are calculated according to the robust Q/Hampel method detailed in International Organization for Standardization (ISO) 13528:2015(E), Annex C. ${ }^{9}$ The robust $\mathrm{Q} /$ Hampel method retains outliers, however their influence declines as their distance from the consensus location increases.

\section{Data Tables}

The data submitted by the participants, listed by lab code, are detailed herein for Control A, Control B, Biomass A, and Biomass B for starch and moisture. Due to the low number of participants reporting results for cellulose/cellulosic content, no data for cellulose and cellulosic content is included. The tables are organized by measurand (starch, moisture), followed by material (Control A, Control B, Biomass A, and Biomass B). The results for all samples of a material (for example Control A-Sample 1 and Control A-Sample 2) are listed in the table along with replicates, if reported. The average and SD for samples with multiple measurements are listed. Community statistics are summarized at the bottom of each sample column, including the consensus mean, robust $\mathrm{SD}$, and the number of participants determined in the analysis. Blank spaces in the data table next to a laboratory indicate that that laboratory did not return data for that analyte-sample combination.

\section{Graphical Representation:}

For each analyte-sample combination, a graphical representation of the data is included and is found below the corresponding data summary table. In this view, individual laboratory data (circles) are plotted with the individual laboratory SDs (rectangles). The green solid line represents the consensus mean and the green shaded area represents the $95 \%$ confidence interval for the consensus mean. The standard uncertainty of the consensus mean is calculated using the equation below (ISO 13528$)^{7}$, based on the robust SD $\left(s^{*}\right.$, listed as the Q/Hampel consensus SD in the data tables) and the number of participants reporting data $(N)$.

$$
u_{\text {mean }}=1.25 \frac{s^{*}}{\sqrt{N}}
$$

The standard uncertainty $\left(u_{\text {mean }}\right)$ is then multiplied by 2 to give an approximate $95 \%$ level of confidence expanded uncertainty $\left(U_{\text {mean }}\right)$.

The blue dotted line, referred to as the reference value, is the gravimetric value, which is only available for Control A and Control B. The pink patterned shaded region represents the expanded uncertainty of the reference value, which was determined by the within-packet and between-packet variability. In this view, the relative locations of individual laboratory data and consensus zones with respect to the reference zone can be compared. The solid red lines represent the range of tolerance $(Z$-score, $|Z| \leq 2)$. Z-scores for this this study were calculated as: 


$$
Z=\frac{x-X_{\text {consensus }}}{s^{*}}
$$

where $x$ is a test result and $X_{\text {consensus }}$ is the consensus mean. The results are color coded based on the method used, a short description of the analytical methods is indicated in the legend of the plot. 


\section{Results and Discussion}

Starch in Control A and Control B:

Control A contained both soluble and resistant starch and was designed to evaluate the suitability of the analytical methods used by the participants to quantify both soluble and resistant starch. Total starch in Control A is estimated as $(67.6 \pm 3.6) \mathrm{cg} / \mathrm{g}$ with a resistant starch content of $13 \mathrm{cg} / \mathrm{g}$.

Table 2 lists the starch content in Control A reported by the participants along with the community results including the consensus mean and the SD of the study. Results are also shown graphically in Figure 2.

Table 2: Data Summary for mass fraction of starch in Control A.

The results, as reported by each laboratory including replicates (when reported) for Samples 1 and 2 , are listed. The community results, including the consensus mean and SD of the interlaboratory study determined by the $\mathrm{Q} /$ Hampel method, are listed along with the median, maximum, and minimum values reported and the number of participants that reported results.

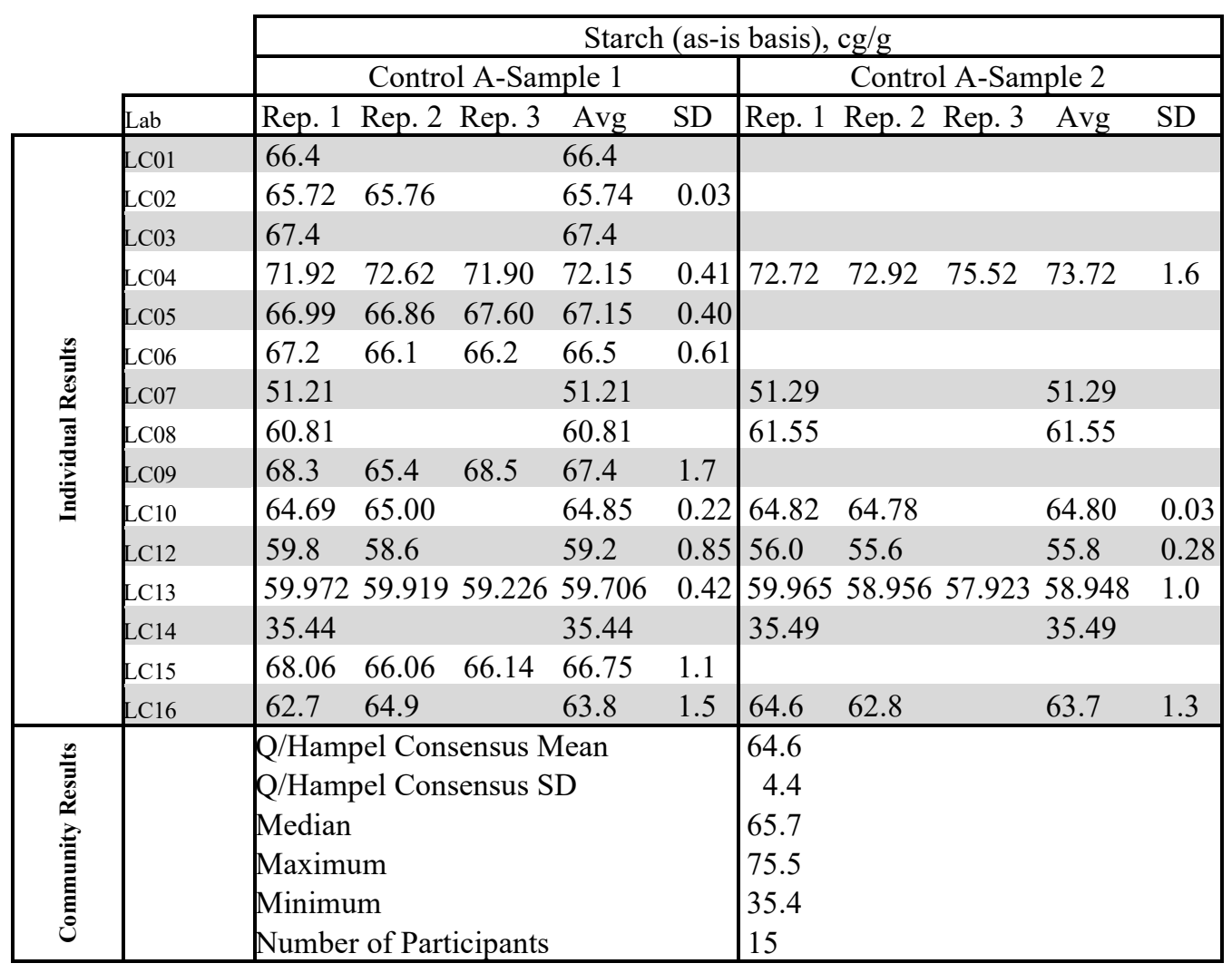




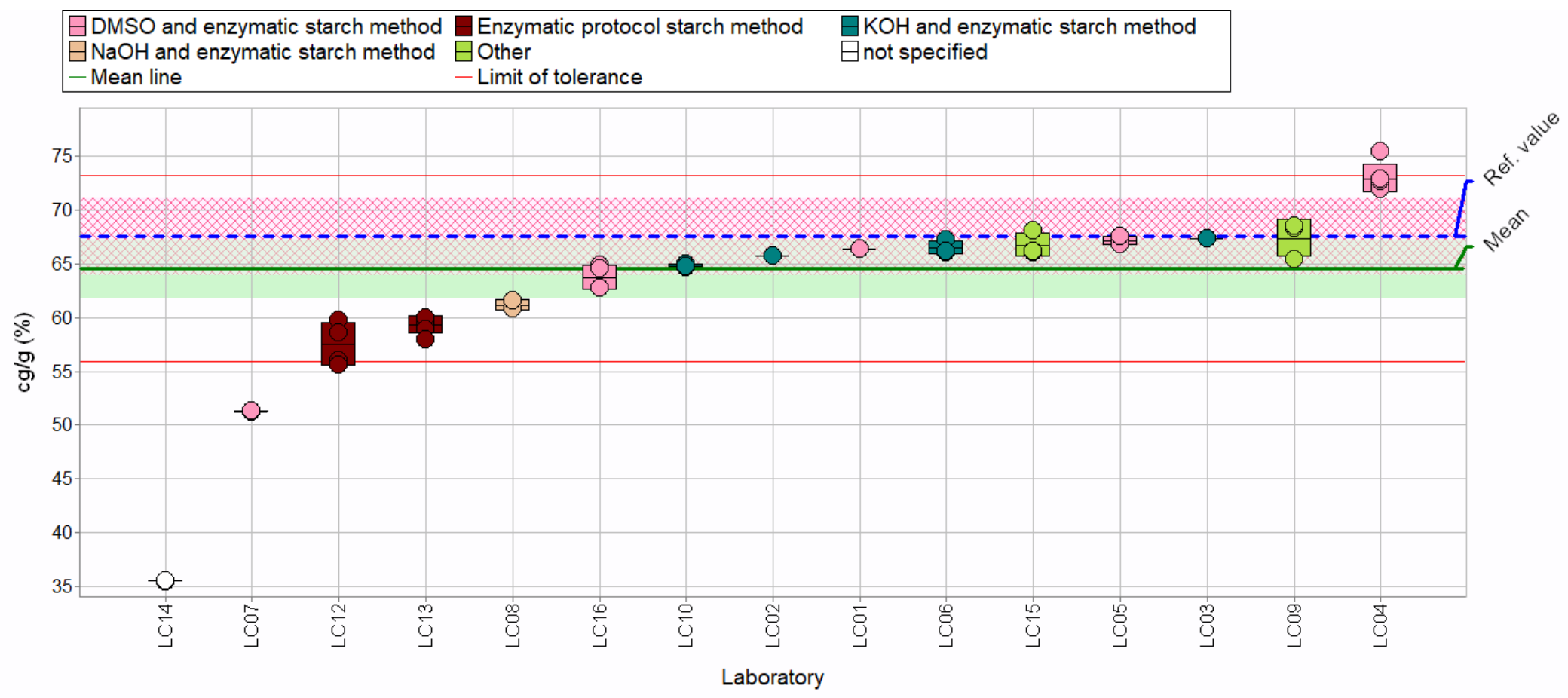

Figure 2: Mass fraction of starch in Control A reported on an as-is basis.

In this view, individual laboratory data are plotted (circles) with individual laboratory SD (rectangle) for all replicates in both Control A-Sample 1 and Control A-Sample 2. The color of the data points represents the method employed by each individual laboratory (see legend). The solid green line represents the consensus mean, and the light green shaded region represents the $95 \%$ confidence interval of the consensus mean. The dotted blue line represents the gravimetric mass fraction, as-is with the red patterned region representing the $95 \%$ confidence interval of the gravimetric value. The red solid lines represent the consensus range of tolerance, calculated as the values above and below the consensus mean that result in $|\mathrm{Z}| \leq 2$. 
The consensus mean (solid green line in Figure 2) for starch in Control A is $4 \%$ low compared to the gravimetric value (blue dotted line in Figure 2). More participants are biased low than biased high relative to the gravimetric value. The presence of resistant starch was clearly a challenge for many analytical protocols used in the interlaboratory study. Upon examining the methods used by the participants (Figure 2 is color coded based on sample preparation method used), all participants that used a $\mathrm{KOH}$ and enzymatic treatment were within the $95 \%$ confidence level of the gravimetric results, indicating that this method can digest resistant starch. The two participants that specified using "other" methods also performed well; unfortunately, no method details are known. There were participants that performed well using DMSO and enzymatic treatment, however, one laboratory was biased high and two participants were biased low using this method.

Method details that were not captured in this study, such as enzyme incubation time, enzyme concentration, agitation, temperature, etc. may be important parameters for successful digestion of both soluble and resistant starch. Results provided by the two participants that used enzymatic protocol without $\mathrm{KOH}, \mathrm{NaOH}$, or DMSO were lower than the gravimetric result. This is compatible with use of a base or solvent being necessary to access the resistant starch.

Ten participants used liquid chromatography (LC) with refractive index as the detection method (see Figure 1b). Laboratories 8, 10, and 14 used spectrophotometric detection, while laboratory 12 used "other" and laboratory 16 did not specify the detection method used. There were no obvious trends based on detection methods used.

A robust SD for the starch content in Control A was estimated as $4.4 \mathrm{cg} / \mathrm{g}$, for a $6.8 \%$ relative standard deviation (RSD). It should be noted that by using the robust $\mathrm{Q} /$ Hampel method, the low results reported by L14 had little to no effect on the consensus mean and SD.

Control B contained soluble starch at a low mass fraction of $(8.98 \pm 0.34) \mathrm{cg} / \mathrm{g}$ with no added resistant starch.

Table 3 shows the results for all replicates of Samples 1 and 2 of Control B. The results are shown graphically in Figure 3. Overall, the community performed better on Control B compared to Control A with the consensus mean being only $0.7 \%$ different from the gravimetric value. It is interesting to note that some participants that got comparable results to the gravimetric value of Control A, were biased high for Control B; this was the case for the two participants that used "other" methods. Participants that used KOH or DMSO with their enzymatic protocols provide similar results. The results from the two participants that used the enzymatic protocol without $\mathrm{KOH}, \mathrm{NaOH}$, or DMSO are also biased low in this control material. A robust SD for the starch content in Control B was estimated as $1.0 \mathrm{cg} / \mathrm{g}$, for a $11 \% \mathrm{RSD}$.

Figure 4 compares the interlaboratory study data returned for Control A and Control B on a single plot. In these types of plots, trends may be emphasized that indicate potential calibration issues or method biases. While Lab 7 and Lab 14 were biased low for Control A they performed well for Control B. Given that Control A contained resistant starch may suggest that the methods used by these two participants do not perform well for the determination of resistant starch. 
Table 3: Data Summary for mass fraction of starch in Control B reported on an as-is basis. The results, as reported by each laboratory including replicates (when reported) for Samples 1 and 2 , are listed. The community results, including the consensus mean and SD of the interlaboratory study determined by the Q/Hampel method, are listed along with the median, maximum, and minimum values reported and the number of participants that reported results.

\begin{tabular}{|c|c|c|c|c|c|c|c|c|c|c|c|}
\hline & \multirow[b]{3}{*}{$\mathrm{Lab}$} & \multicolumn{10}{|c|}{ Starch (as-is basis), cg/g } \\
\hline & & \multicolumn{5}{|c|}{ Control B-Sample 1} & \multicolumn{5}{|c|}{ Control B-Sample 2} \\
\hline & & Rep. 1 & Rep. 2 & Rep. 3 & Avg & $\mathrm{SD}$ & Rep. 1 & Rep. 2 & Rep. 3 & Avg & $\mathrm{SD}$ \\
\hline \multirow{15}{*}{ 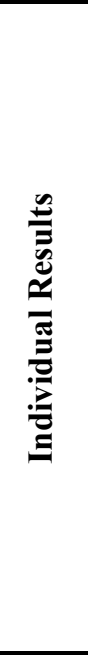 } & LC01 & 8.7 & & & 8.7 & & & & & & \\
\hline & LC02 & 8.64 & 8.74 & & 8.69 & 0.07 & & & & & \\
\hline & LC03 & 10.3 & & & 10.3 & & & & & & \\
\hline & LC04 & 9.19 & 9.81 & 9.59 & 9.53 & 0.31 & 9.73 & 9.68 & 9.92 & 9.78 & 0.13 \\
\hline & LC05 & 8.94 & 8.83 & 9.06 & 8.94 & 0.12 & & & & & \\
\hline & LC06 & 9.42 & 8.92 & 8.90 & 9.08 & 0.30 & & & & & \\
\hline & LC07 & 8.17 & & & 8.17 & & 8.44 & & & 8.44 & \\
\hline & LC08 & 8.06 & & & 8.06 & & 7.97 & & & 7.97 & \\
\hline & LC09 & 11.1 & 11.1 & 11.5 & 11.2 & 0.23 & & & & & \\
\hline & LC10 & 9.10 & 8.92 & & 9.01 & 0.13 & 8.89 & 8.83 & & 8.86 & 0.04 \\
\hline & LC12 & 7.47 & 7.67 & & 7.57 & 0.14 & 7.85 & 7.66 & & 7.76 & 0.13 \\
\hline & LC13 & 8.207 & 8.173 & 6.697 & 7.692 & 0.86 & 8.556 & 8.527 & 6.946 & 8.010 & 0.92 \\
\hline & LC14 & 8.14 & & & 8.14 & & 8.00 & & & 8.00 & \\
\hline & LC15 & 9.97 & 11.06 & 9.88 & 10.30 & 0.66 & & & & & \\
\hline & LC16 & 8.86 & 8.88 & & 8.87 & 0.02 & 8.80 & 8.77 & & 8.79 & 0.02 \\
\hline 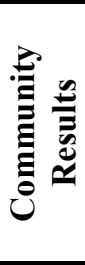 & & $\begin{array}{l}\text { Q/Ham } \\
\text { Q/Ham } \\
\text { Median } \\
\text { Maxim } \\
\text { Minimu } \\
\text { Numbe }\end{array}$ & $\begin{array}{l}\text { pel Con } \\
\text { pel Con } \\
\text { um } \\
\text { um of Par }\end{array}$ & $\begin{array}{l}\text { sensus } 1 \\
\text { sensus } \\
\text { ticipants }\end{array}$ & $\begin{array}{l}\text { Mean } \\
\text { SD }\end{array}$ & & \begin{tabular}{|c|}
8.92 \\
1.0 \\
8.83 \\
11.5 \\
6.70 \\
15 \\
\end{tabular} & & & & \\
\hline
\end{tabular}




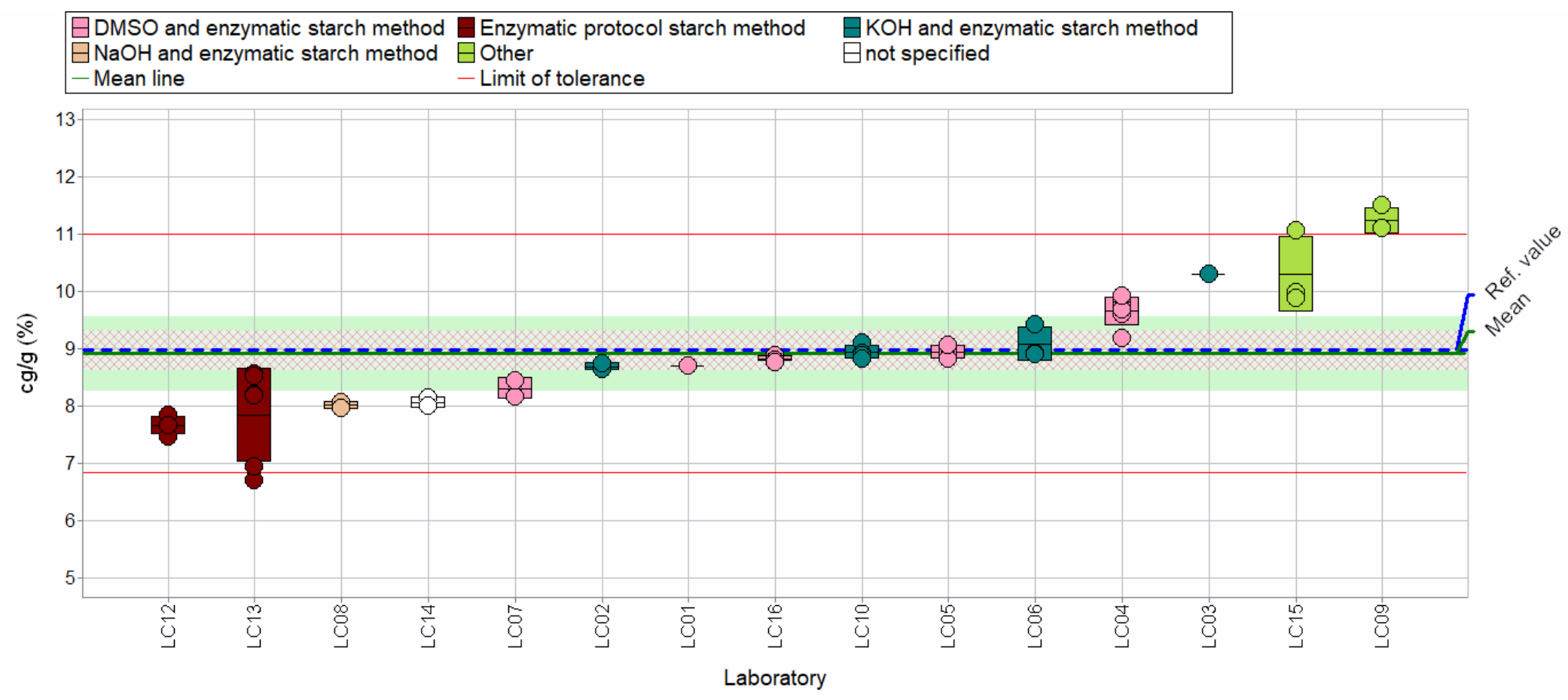

Figure 3: Mass fraction of starch in Control B reported on an as-is basis.

In this view, individual laboratory data are plotted (circles) with individual laboratory SD (rectangle) for all replicates in both Control B-Sample 1 and Control B-Sample 2. The color of the data points represents the method employed by each individual laboratory (see legend). The solid green line represents the consensus mean, and the light green shaded region represents the $95 \%$ confidence interval of the consensus mean. The dotted blue line represents the gravimetric mass fraction of starch, as-is with the red patterned region representing the $95 \%$ confidence interval of the gravimetric value. The red solid lines represent the consensus range of tolerance, calculated as the values above and below the consensus mean that result in $|\mathrm{Z}| \leq 2$. 


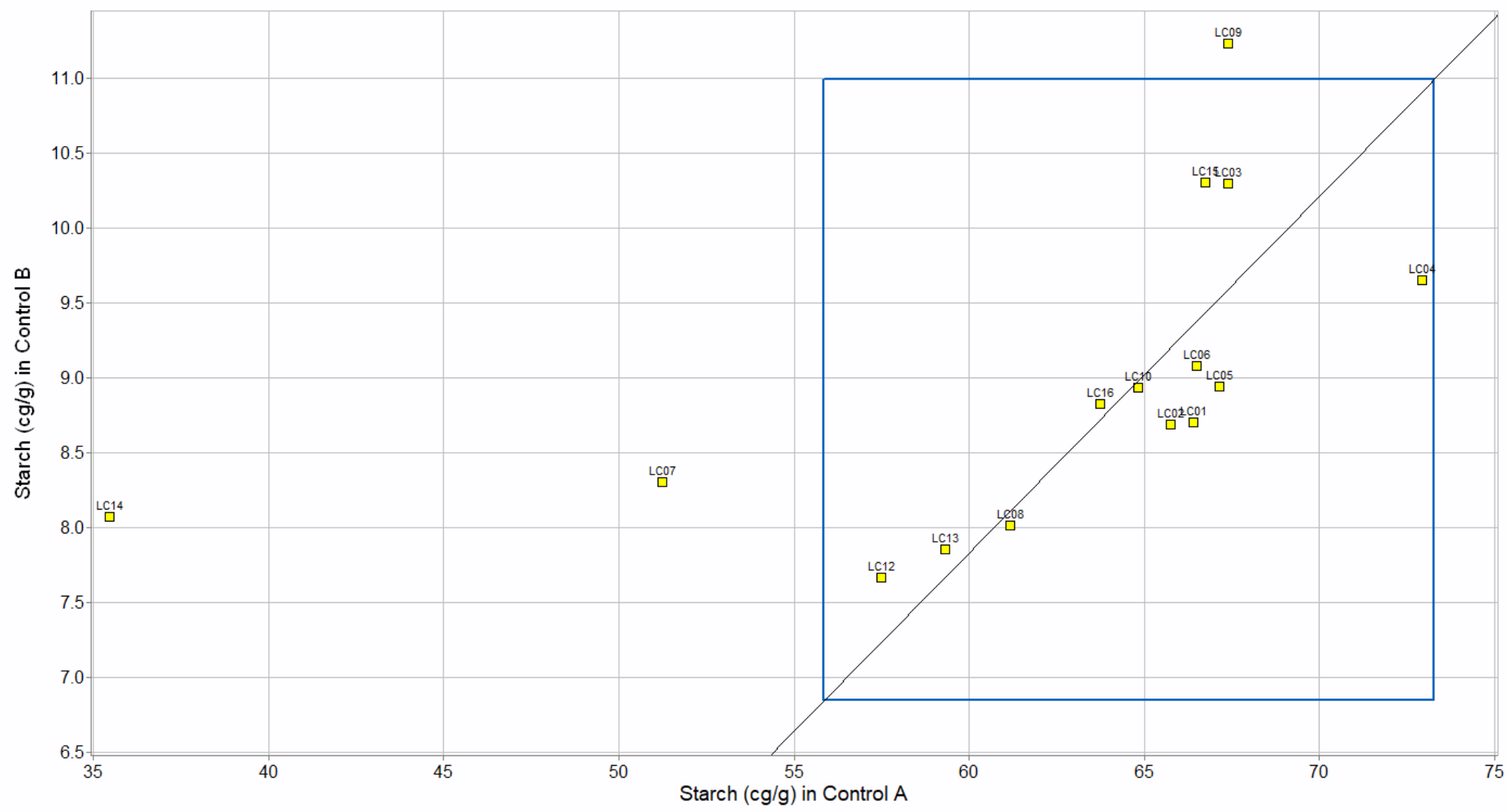

Figure 4: Laboratory means for mass fraction of starch in Control A and in Control B.

In this view (sample/sample comparison view), the individual laboratory means for one sample (Control A) is compared to the individual laboratory means for a second sample (Control B). The solid blue box represents the consensus range of tolerance for Control A (x-axis) and Control B (y-axis), calculated as the values above and below the consensus means that result in $|\mathrm{Z}| \leq 2$. The black line is an angular bisector through the data to help visualize if there is a correlation of results between the two samples. 
Starch in Biomass A and Biomass B:

Biomass A, a candidate RM, is a dried corn grain biomass intermediate before conversion, obtained from a commercial ethanol plant and is representative of routine samples. The true value of starch content in this material is unknown. Table 4 lists the results for all replicates of Samples 1 to 4 of Biomass A; the results are shown graphically in Figure 5 .

The range of total starch reported for Biomass A is small compared to Control A. A robust SD of $3.8 \mathrm{cg} / \mathrm{g}$, RSD of $5.8 \%$, was estimated for the percent starch in Biomass A. This is slightly improved from Control A. The reduced variability of Biomass A compared to Control A is most likely due to the lower amount of resistant starch present in Biomass A. While Biomass A was dried at high temperatures to create some resistant starch, this material does not nearly contain as much compared to Control A.

Most methods seem to perform reasonably well for this material; however, it is difficult to make definite conclusions because the "true" starch value of this material is unknown.

Biomass $\mathrm{B}$, a candidate RM, is a dried corn grain biomass intermediate after conversion obtained from a commercial ethanol plant. Table 5 lists the results for all replicates of Samples 1-4 of Biomass B. The results are also shown graphically in Figure 6.

For Biomass B, similar trends are observed when compared to Control B, where the two participants that used "other" methods reported higher results compared to the other participants. The robust SD of the study for Biomass B was $1.5 \mathrm{cg} / \mathrm{g}$, for an RSD of $43 \%$. The large RSD is partially attributable to the low amount of starch that is estimated for the material.

Figure 7 compares the percent starch determined in Biomass A and Biomass B. There are no results that deviate widely from the angular bisector line (diagonal black line in Figure 7) which is the line produced from points of identical z-score in both samples. This implies that the methods used do not bias one material over the other (this was not the case in Figure 4). The correlation of results along the bisector suggests calibration differences among the participating laboratories. It is expected that there would be less variability/dispersion in the data if a common calibration method and common calibrants were used by all participants. For this study, no calibration details were recorded by the participants. 
Table 4: Data Summary for mass fraction of starch in Biomass A. The results, as reported by each laboratory including replicates (when reported) for Samples 1-4, are listed. The community results, including the consensus mean and SD of the interlaboratory study determined by the $\mathrm{Q} /$ Hampel method, are listed along with the median, maximum, and minimum values reported and the number of participants that reported results.

\begin{tabular}{|c|c|c|c|c|c|c|c|c|c|c|c|c|c|c|c|c|c|c|c|c|c|}
\hline & & \multicolumn{20}{|c|}{ Starch (as-is basis), cg/g } \\
\hline & & \multicolumn{5}{|c|}{ Biomass A-Sample 1} & \multicolumn{5}{|c|}{ Biomass A-Sample 2} & \multicolumn{5}{|c|}{ Biomass A-Sample 3} & \multicolumn{5}{|c|}{ Biomass A-Sample 4} \\
\hline & $\mathrm{Lab}$ & Rep. 1 & Rep. 2 & Rep. 3 & Avg & SD & Rep. 1 & Rep. 2 & Rep. 3 & Avg & SD & Rep. 1 & Rep. 2 & Rep. 3 & Avg & SD & Rep. 1 & Rep. 2 & Rep. 3 & Avg & SD \\
\hline \multirow{15}{*}{ 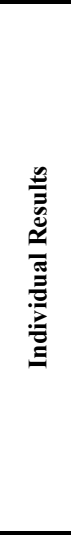 } & LC01 & 66.1 & & & 66.1 & & 65.9 & & & 65.9 & & 65.9 & & & 65.9 & & & & & & \\
\hline & LC02 & 65.44 & 65.59 & & 65.52 & 0.11 & 65.45 & 66.00 & & 65.73 & 0.39 & 65.96 & 65.81 & & 65.89 & 0.11 & & & & & \\
\hline & $\mathrm{LC} 03$ & 69.2 & & & 69.2 & & 68.8 & & & 68.8 & & 66.6 & & & 66.60 & & & & & & \\
\hline & LC04 & 71.39 & 71.13 & 71.76 & 71.43 & 0.32 & 70.98 & 71.19 & 71.12 & 71.10 & 0.11 & 71.29 & 71.50 & 71.07 & 71.29 & 0.22 & & & & & \\
\hline & LC05 & 67.82 & 68.61 & 68.85 & 68.43 & 0.54 & 67.91 & 68.44 & 68.79 & 68.38 & 0.44 & 68.28 & 69.05 & 69.19 & 68.84 & 0.49 & & & & & \\
\hline & LC06 & 67.1 & 66.7 & 62.6 & 65.5 & 2.5 & 67.3 & 66.7 & 67.1 & 67.0 & 0.31 & 66.1 & 66.7 & 66.1 & 66.3 & 0.35 & & & & & \\
\hline & LC07 & 65.28 & & & 65.28 & & 65.70 & & & 65.70 & & 65.02 & & & 65.02 & & 65.53 & & & 65.53 & \\
\hline & LC08 & 60.75 & & & 60.75 & & 60.63 & & & 60.63 & & 60.81 & & & 60.81 & & 61.15 & & & 61.15 & \\
\hline & LC09 & 72.2 & 68.6 & 70.1 & 70.3 & 1.8 & 67.4 & 71.2 & 67.5 & 68.7 & 2.2 & 70.5 & 68.5 & 72.0 & 70.3 & 1.8 & & & & & \\
\hline & LC10 & 63.11 & 62.83 & 63.34 & 63.09 & 0.26 & 63.87 & 63.59 & 63.33 & 63.60 & 0.27 & 63.39 & 63.75 & 63.22 & 63.45 & 0.27 & 63.58 & 63.40 & 63.84 & 63.61 & 0.22 \\
\hline & LC12 & 63.4 & 64.2 & & 63.8 & 0.57 & 62.3 & 60.70 & & 61.5 & 1.1 & 60.4 & 59.4 & & 59.9 & 0.71 & & & & & \\
\hline & LC13 & 62.815 & 62.336 & 62.375 & 62.509 & 0.27 & 62.098 & 62.157 & 62.163 & 62.139 & 0.04 & 62.042 & 62.817 & 61.328 & 62.062 & 0.75 & 62.149 & 62.564 & 62.514 & 62.409 & 0.23 \\
\hline & LC14 & 64.50 & & & 64.50 & & 65.77 & & & 65.77 & & 65.77 & & & 65.77 & & 65.48 & & & 65.48 & \\
\hline & LC15 & 66.20 & 68.89 & 67.77 & 67.62 & 1.4 & 68.75 & 67.91 & 67.72 & 68.13 & 0.55 & 66.53 & 67.84 & 68.03 & 67.47 & 0.82 & & & & & \\
\hline & LC16 & 61.8 & 62.4 & & 62.1 & 0.41 & & & & & & & & & & & & & & & \\
\hline 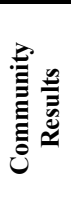 & & $\begin{array}{l}\mathrm{Q} / \text { Hamp } \\
\mathrm{Q} / \text { Hamp } \\
\text { Median } \\
\text { Maximu } \\
\text { Minimun } \\
\text { Number }\end{array}$ & $\begin{array}{l}\text { el Consen } \\
\text { el Consen } \\
\text { of Particit }\end{array}$ & $\begin{array}{l}\text { us Mean } \\
\text { us SD } \\
\text { ants }\end{array}$ & & & & & & & & $\begin{array}{r}65.6 \\
3.8 \\
65.7 \\
72.2 \\
59.4 \\
15\end{array}$ & & & & & & & & & \\
\hline
\end{tabular}




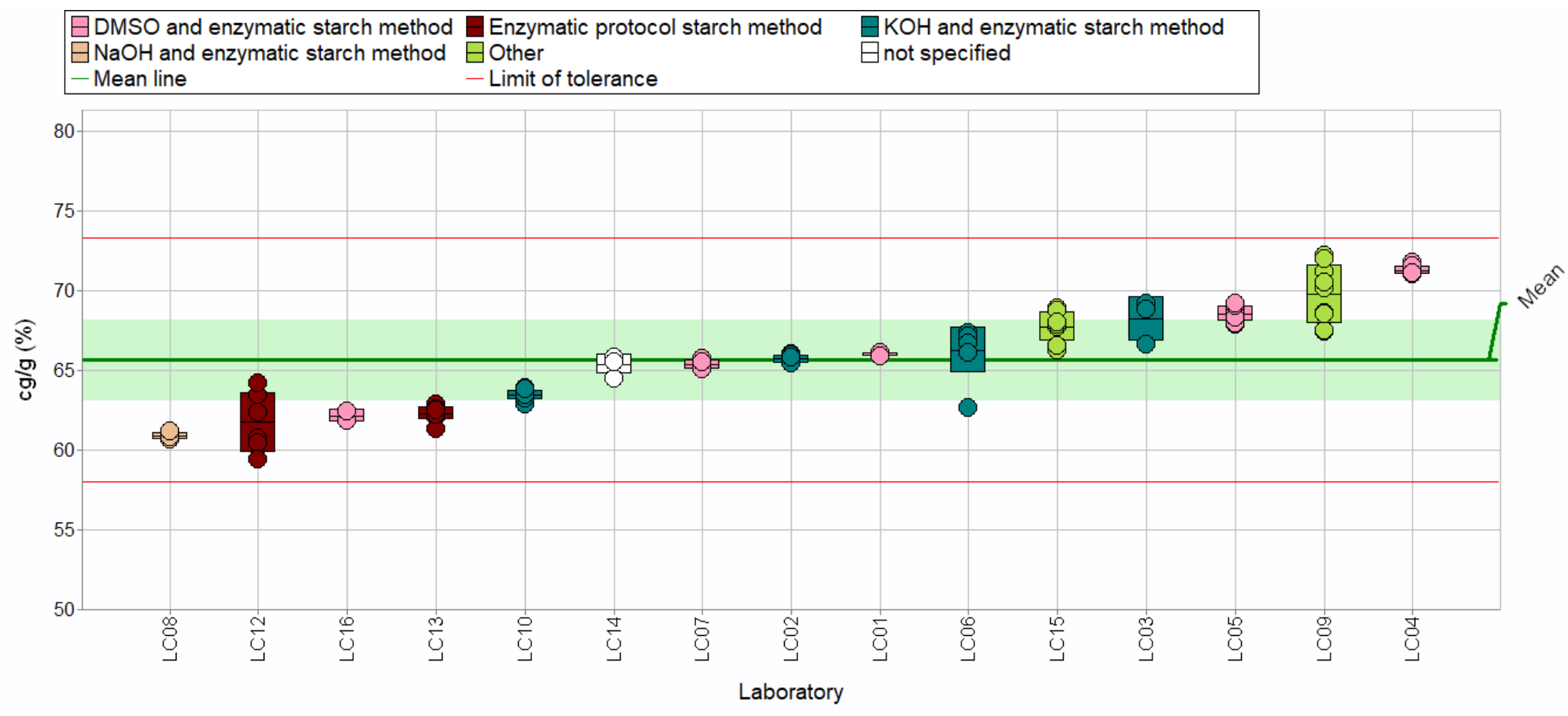

Figure 5: Mass fraction of starch in Biomass A reported on an as-is basis.

In this view, individual laboratory data are plotted (circles) with individual laboratory SD (rectangle) for all replicates in Biomass ASample 1, Sample 2, Sample 3, and Sample 4. The color of the data points represents the method employed by each individual laboratory (see legend). The solid green line represents the consensus mean, and the light green shaded region represents the $95 \%$ confidence interval of the consensus mean. The red solid lines represent the consensus range of tolerance, calculated as the values above and below the consensus mean that result in $|\mathrm{Z}| \leq 2$. 
Table 5: Data Summary for the mass fraction of starch in Biomass B.

The results, as reported by each laboratory including replicates (when reported) for Samples 1-4, are listed. The community results, including the consensus mean and SD of the interlaboratory study determined by the Q/Hampel method, are listed along with the median, maximum, and minimum values reported and the number of participants that reported results.

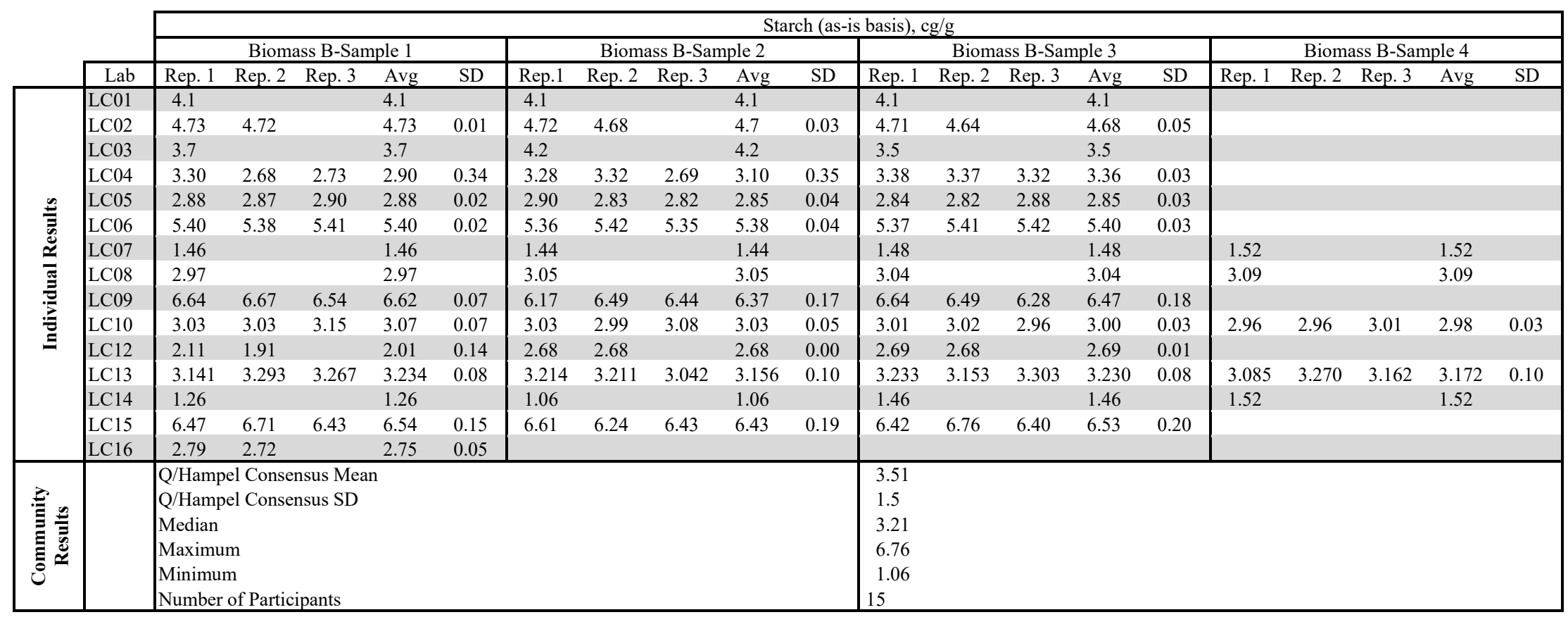




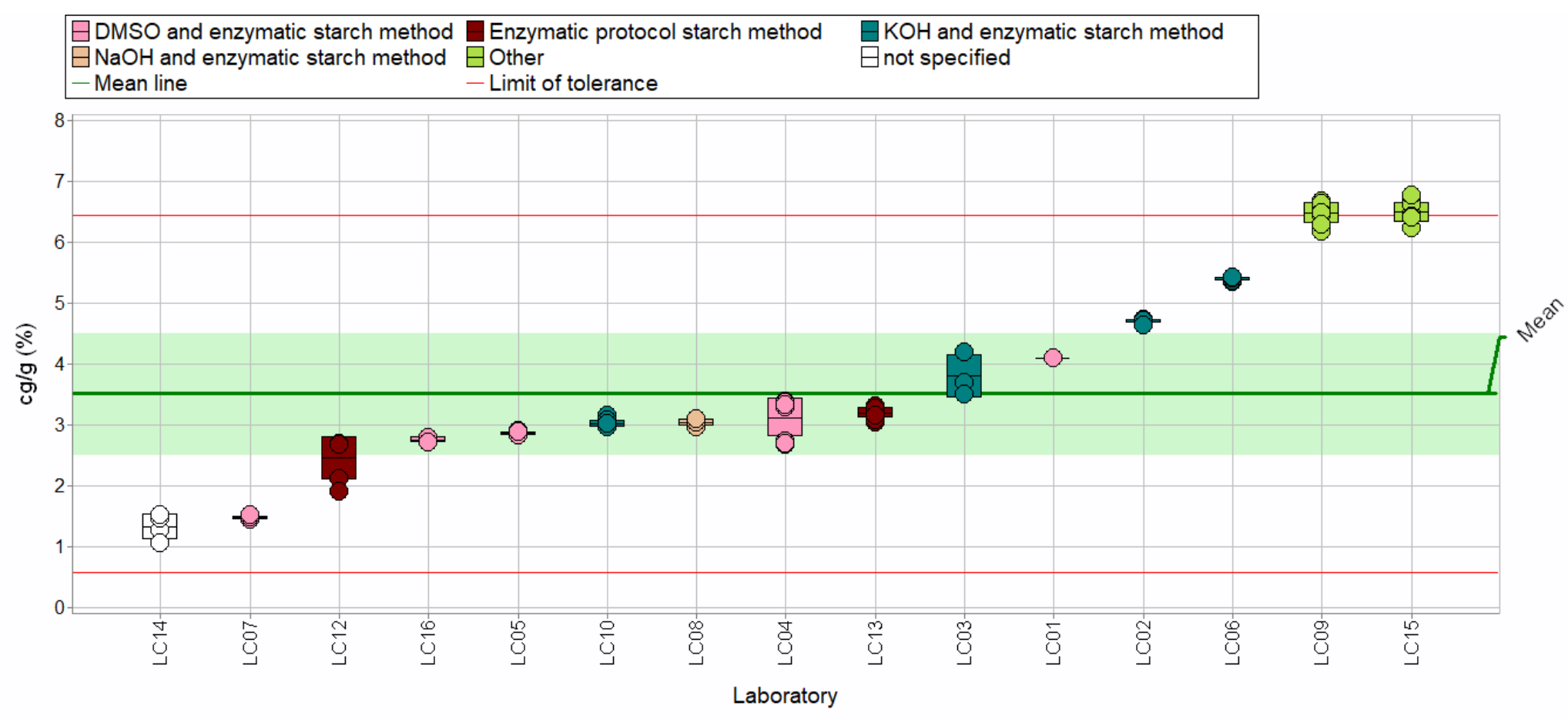

Figure 6: Mass fraction of starch in Biomass B reported on an as-is basis.

In this view, individual laboratory data are plotted (circles) with individual laboratory SD (rectangle) for all replicates in Biomass BSample 1, Sample 2, Sample 3, and Sample 4. The color of the data points represents the method employed by each individual laboratory (see legend). The solid green line represents the consensus mean, and the light green shaded region represents the $95 \%$ confidence interval of the consensus mean. The red solid lines represent the consensus range of tolerance, calculated as the values above and below the consensus mean that result in $|Z| \leq 2$. 


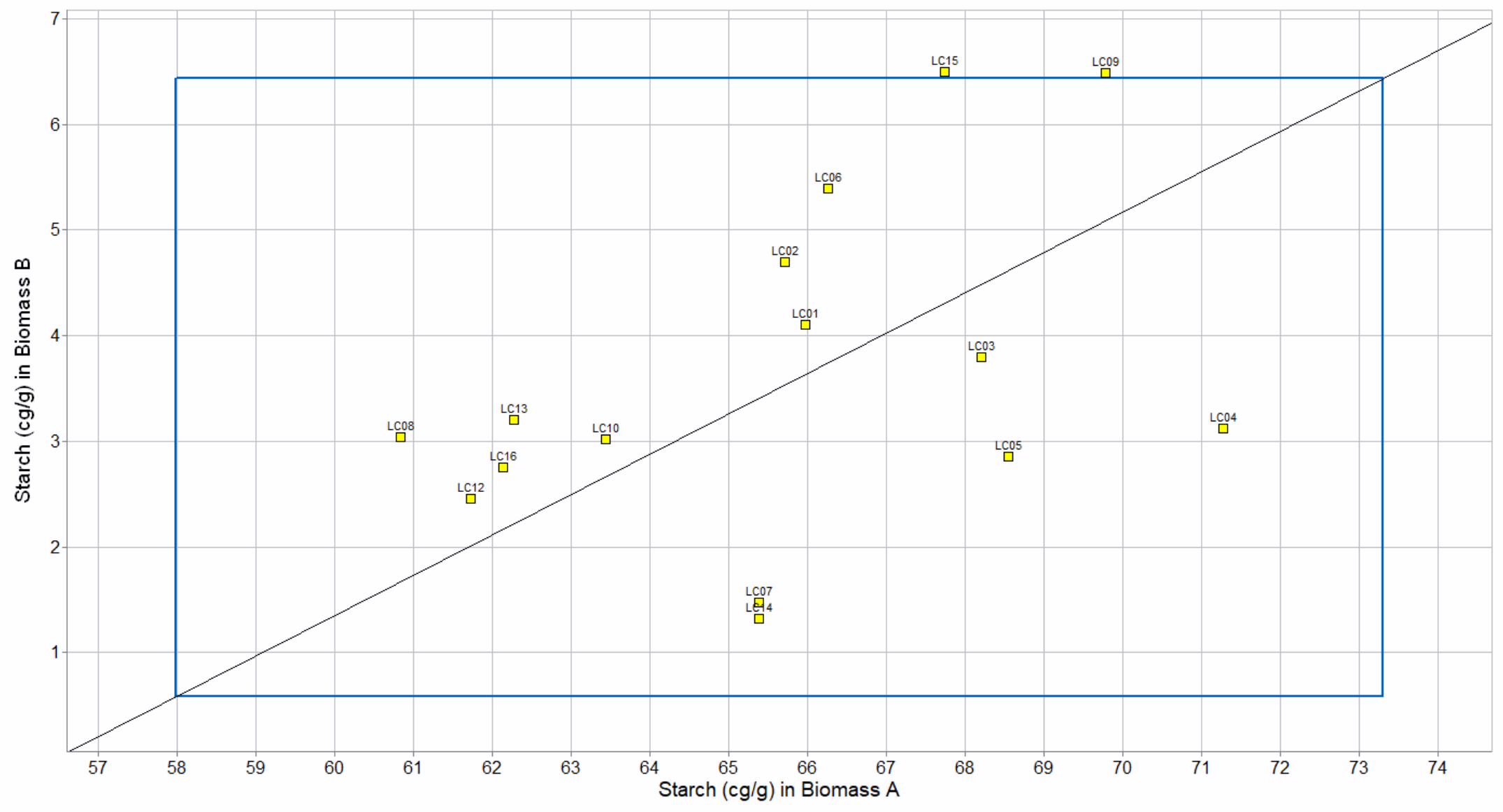

Figure 7: Laboratory means of the mass fraction of starch in Biomass A and in Biomass B.

In this view (sample/sample comparison view), the individual laboratory means for one sample (Biomass A) is compared to the individual laboratory means for a second sample (Biomass B). The solid blue box represents the consensus range of tolerance for Biomass A (x-axis) and Biomass B (y-axis), calculated as the values above and below the consensus means that result in $|\mathrm{Z}| \leq 2$. The black line is an angular bisector through the data to help visualize if there is a correlation of results between the two samples. 


\section{Within-laboratory variability:}

The within-laboratory variability can be accessed by examining the results from each laboratory from different samples and replicates of the same material. For example, Biomass A-Sample 1, Biomass A-Sample 2, and Biomass A-Sample 3, are the same material that is packaged in separate bags; the variability of each laboratory can be evaluated by comparing the results from Biomass A Sample 1-3, even if a laboratory only reported one replicate for each sample. Table 6 lists the RSD for all reported measurements of the same material, including replicates, for all the participants in the study. The RSDs are low for most participants, suggesting the participants were able to make precise measurements within their laboratory. This is important when evaluating candidate RMs; the methods used by participants can achieve low SDs among replicates of the complex solid candidate RMs.

Table 6: RSD for all replicates reported by each lab for each material.

\begin{tabular}{|c|c|c|c|c|c|c|c|c|}
\cline { 2 - 8 } \multicolumn{1}{c|}{} & \multicolumn{2}{c|}{ Control A } & \multicolumn{2}{c|}{ Control B } & \multicolumn{2}{c|}{ Biomass A } & \multicolumn{2}{c|}{ Biomass B } \\
\hline Lab & RSD $^{\mathrm{a}}$ & $N^{\mathrm{b}}$ & RSD $^{\mathrm{a}}$ & $N^{\mathrm{b}}$ & $\mathrm{RSD}^{\mathrm{a}}$ & $N^{\mathrm{b}}$ & RSD $^{\mathrm{a}}$ & $N^{\mathrm{b}}$ \\
\hline LC01 & & 1 & & 1 & 0.18 & 3 & 0.0 & 3 \\
LC02 & 0.04 & 2 & 0.8 & 2 & 0.38 & 6 & 0.7 & 6 \\
LC03 & & 1 & & 1 & 2.1 & 3 & 9.5 & 3 \\
LC04 & 1.83 & 6 & 2.6 & 6 & 0.34 & 9 & 10 & 9 \\
LC05 & 0.59 & 3 & 1.3 & 3 & 0.70 & 9 & 1.1 & 9 \\
LC06 & 0.91 & 3 & 3.2 & 3 & 2.2 & 9 & 0.5 & 9 \\
LC07 & 0.11 & 2 & 2.3 & 2 & 0.45 & 3 & 2.3 & 3 \\
LC08 & 0.86 & 2 & 0.8 & 2 & 0.37 & 3 & 1.6 & 3 \\
LC09 & 2.57 & 3 & 2.1 & 3 & 2.6 & 9 & 2.6 & 9 \\
LC10 & 0.20 & 4 & 1.3 & 4 & 0.48 & 9 & 1.8 & 9 \\
LC12 & 3.53 & 4 & 2.0 & 4 & 3.0 & 6 & 14 & 6 \\
LC13 & 1.37 & 6 & 10 & 6 & 0.64 & 9 & 2.6 & 9 \\
LC14 & 0.10 & 2 & 1.2 & 2 & 0.92 & 3 & 16 & 3 \\
LC15 & 1.70 & 3 & 6.4 & 3 & 1.3 & 9 & 2.5 & 9 \\
LC16 & 1.83 & 4 & 0.6 & 4 & 0.66 & 2 & 1.8 & 2 \\
\hline
\end{tabular}

a Relative SD expressed as a percent

b Number of replicates

Within-and Between-Sample Variability for Starch Analysis:

Because many participants reported replicates from multiple samples, the within- and betweensample SDs can be compared for each material. This is valuable for evaluating candidate RMs since the comparison can provide further insight into the homogeneity of the materials. For example, a larger between-sample variation (the variation when analyzing from multiple packets) compared to the within-sample variation (the variation when analyzing multiple samples from the same packet) can indicate that the material is not homogenous between packets. Large withinsample variability may also indicate that the material in a single packet may not be homogenous and may require mixing before sampling or require larger sample sizes to improve the variability.

Figure 8 displays the between-sample RSD as a function of the within-sample RSD for all four materials for participants that reported replicates for two or more samples (packets). The betweensample variability is relatively low $(<5 \%)$ for most materials. Laboratory 12 reported higher between-sample variability compared to within-sample variability for three out of the four 
materials. This was the only laboratory that reported results with samples of the same material that ANOVA determined to be statistically different (for three out of four materials). This could be a result of differences between packets that were analyzed by this laboratory or of differences in laboratory protocol used for different samples. It is possible that one of the participants received a sample that differed from the other samples. In the case of Laboratory 12 , this is unlikely since this laboratory reported differences among samples for three out of four materials. It is more likely that the between-sample differences reported by laboratory 12 are due to slight differences in protocols used when analyzing different samples of the same material, for instance using a different analyst for each sample.

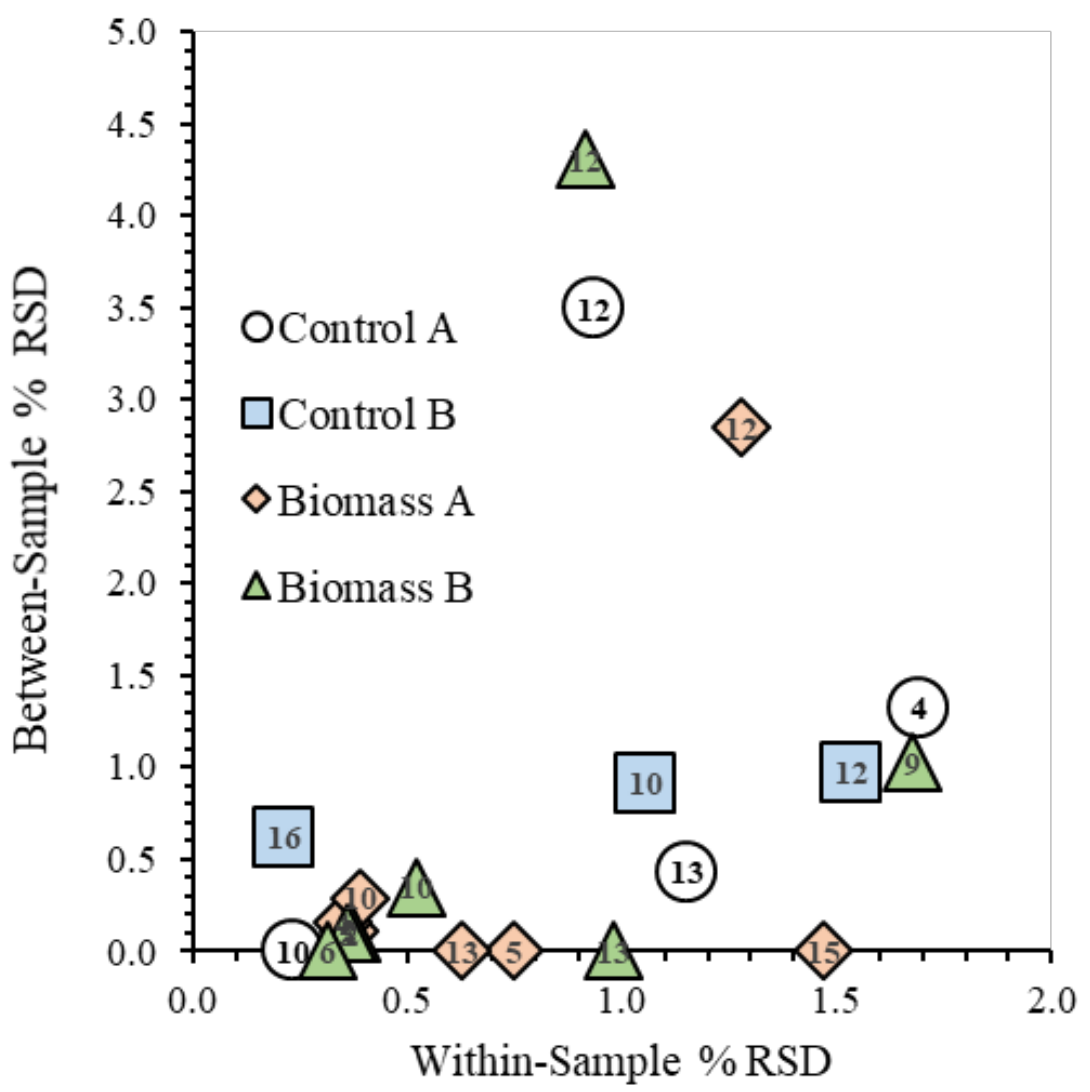

Figure 8: Between-Sample Variability as a function of Within-Sample Variability The symbols represent the estimated within- and between-sample RSDs for each participant that provided replicate information in multiple samples for the four materialsControl A, Control B, Biomass A, and Biomass B. Participants are identified by their lab code numbers.

Figure 8 also shows that three participants reported an approximate between-sample RSD of $1 \%$ for Control B. This suggest that there may be $1 \%$ sample to sample difference for Control B. All other materials appear to be relatively homogenous.

Moisture Content:

Reporting moisture content in this interlaboratory study was optional. Participants were advised to use a forced oven drying protocol as detailed in AOAC 935.29 or NFTA 2.2.2.5. The moisture 
content, as reported by the participants, is listed in Tables 7 to 10 for Control A, Control B, Biomass A and Biomass B, respectively. The data is shown graphically in Figures 9 to 12.

Overall, there is good agreement between all participants that reported moisture for Control A and B. The RSD for Control A and B is $7.9 \%$ and $13 \%$, respectively. The RSD for moisture content for Biomass A and Biomass B is $6.4 \%$ and $25 \%$, respectively. The highest variability of moisture was for Biomass B.

While very similar results were reported among participants that used NFTA 2.2.2.5 and among those that used AOAC 935.29, the AOAC results are consistently smaller than the NFTA. Two participants that used "other" methods reported low values for both Control A and B and high values for Biomass $\mathrm{A}$ and $\mathrm{B}$.

Table 7: Data summary for the mass fraction of moisture in Control A.

Individual laboratory results, as reported for Samples 1 and 2, are listed. The community results, including the consensus mean and SD of the interlaboratory study determined by the Q/Hampel method, are listed along with the median, maximum, and minimum values reported and the number of participants that reported results.

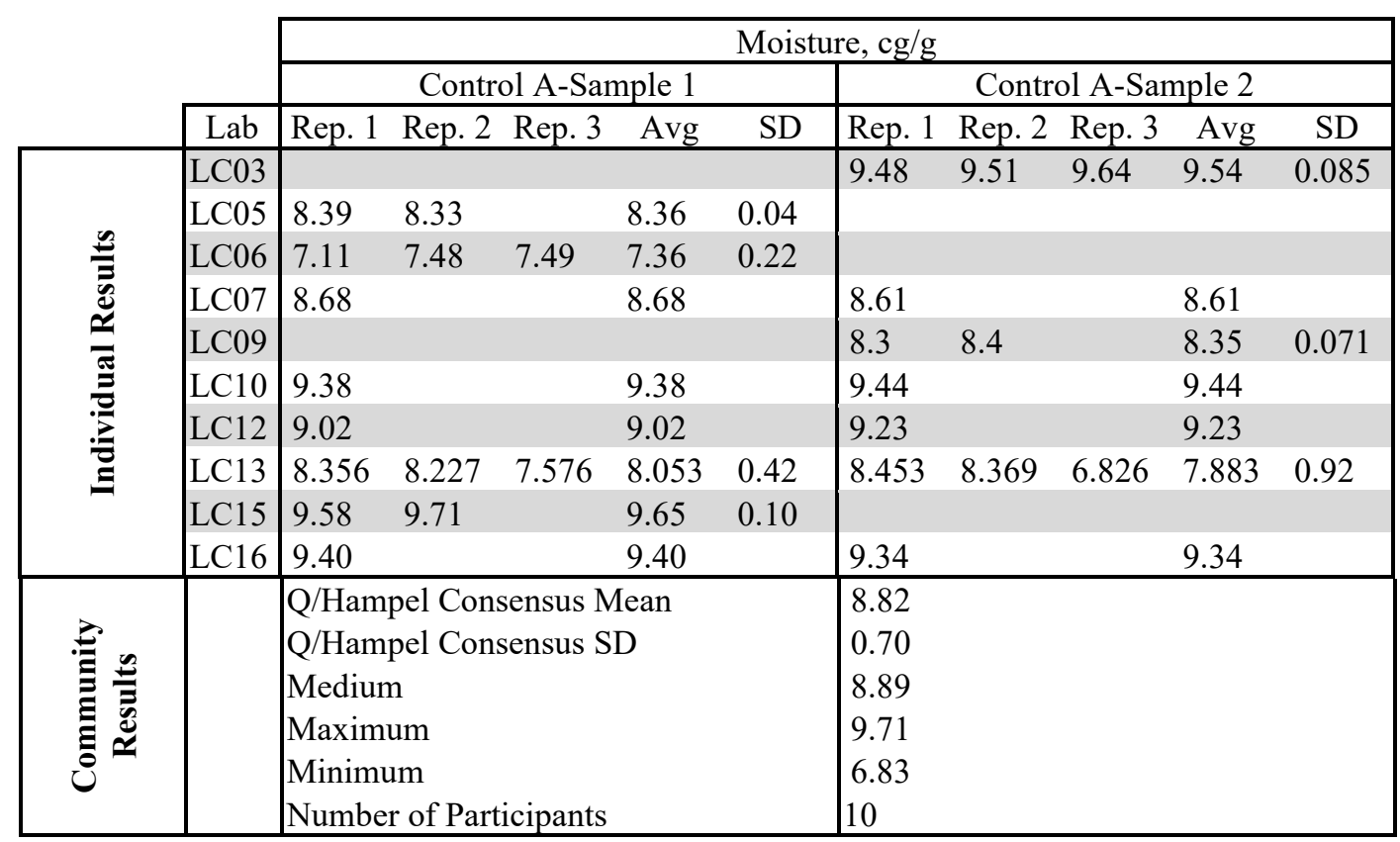




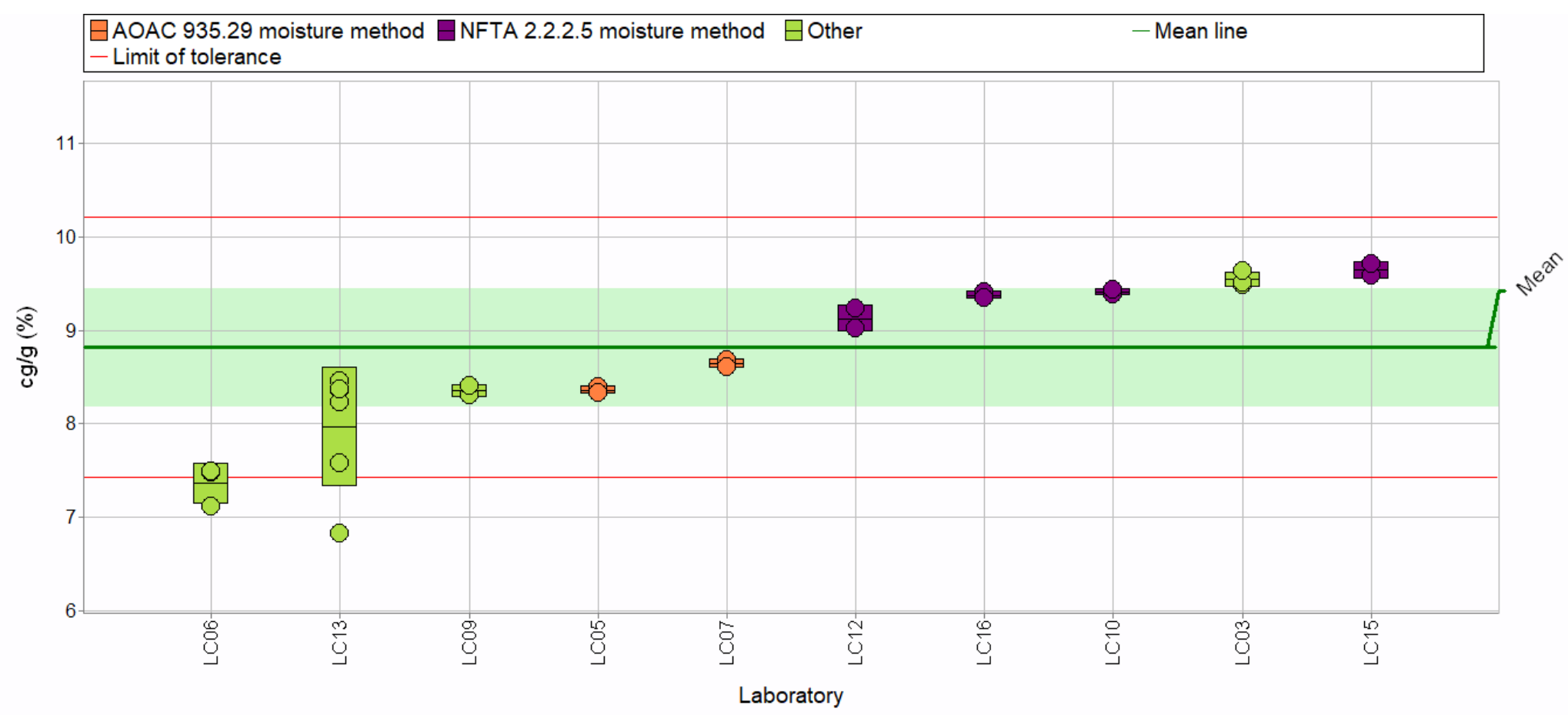

Figure 9: Mass fraction of moisture content in Control A.In this view, individual laboratory data are plotted (circles) with individual laboratory SD (rectangle) for all replicates in Control A-Sample 1 and Sample 2. The color of the data points represents the method employed by each individual laboratory (see legend). The solid green line represents the consensus mean, and the light green shaded region represents the $95 \%$ confidence interval of the consensus mean. The red solid lines represent the consensus range of tolerance, calculated as the values above and below the consensus mean that result in $|Z| \leq 2$. 
Table 8: Data summary for mass fraction of moisture in Control B.Individual laboratory results, as reported for Samples 1 and 2, are listed. The community results, including the consensus mean and SD of the interlaboratory study determined by the Q/Hampel method, are listed along with the median, maximum, and minimum values reported and the number of participants that reported results.

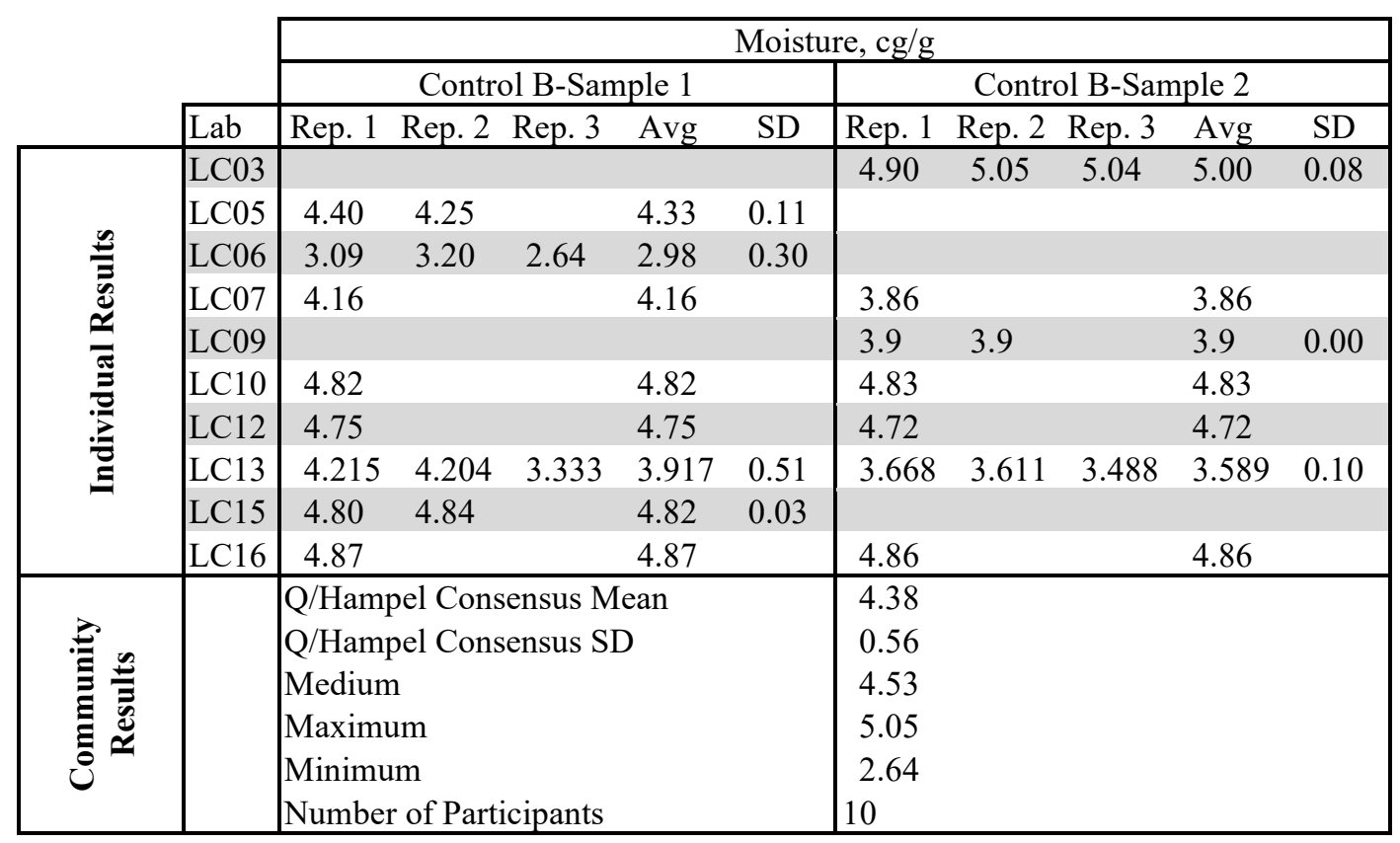




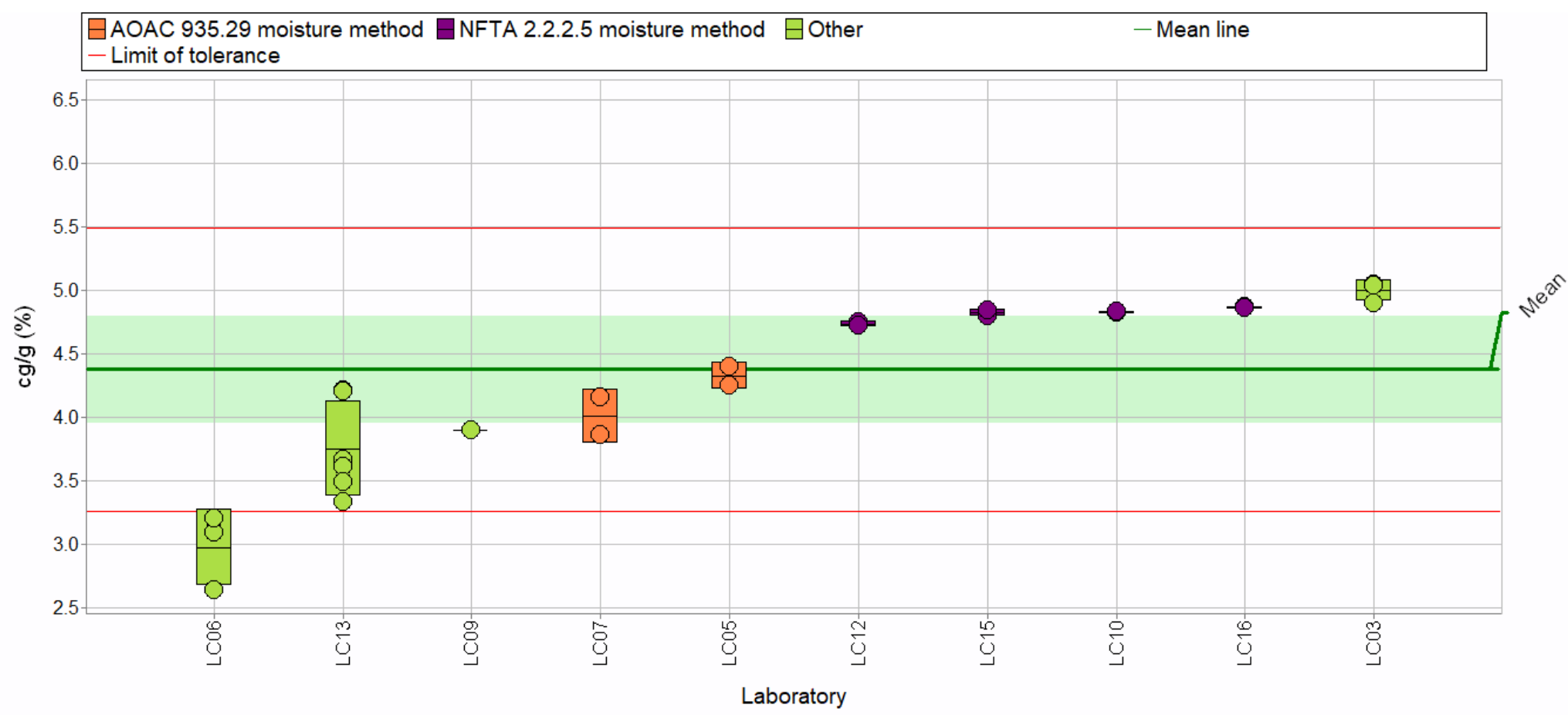

Figure 10: Mass fraction of moisture content in Control B.

In this view, individual laboratory data are plotted (circles) with individual laboratory SD (rectangle) for all replicates in Control BSample 1 and Sample 2. The color of the data points represents the method employed by each individual laboratory (see legend). The solid green line represents the consensus mean, and the light green shaded region represents the $95 \%$ confidence interval of the consensus mean. The red solid lines represent the consensus range of tolerance, calculated as the values above and below the consensus mean that result in $|\mathrm{Z}| \leq 2$. 
Table 9: Data summary for mass fraction of moisture in Biomass A.Individual laboratory results, as reported for Samples 1-4, are listed. The community results, including the consensus mean and SD of the interlaboratory study determined by the Q/Hampel method, are listed along with the median, maximum, and minimum values reported and the number of participants that reported results.

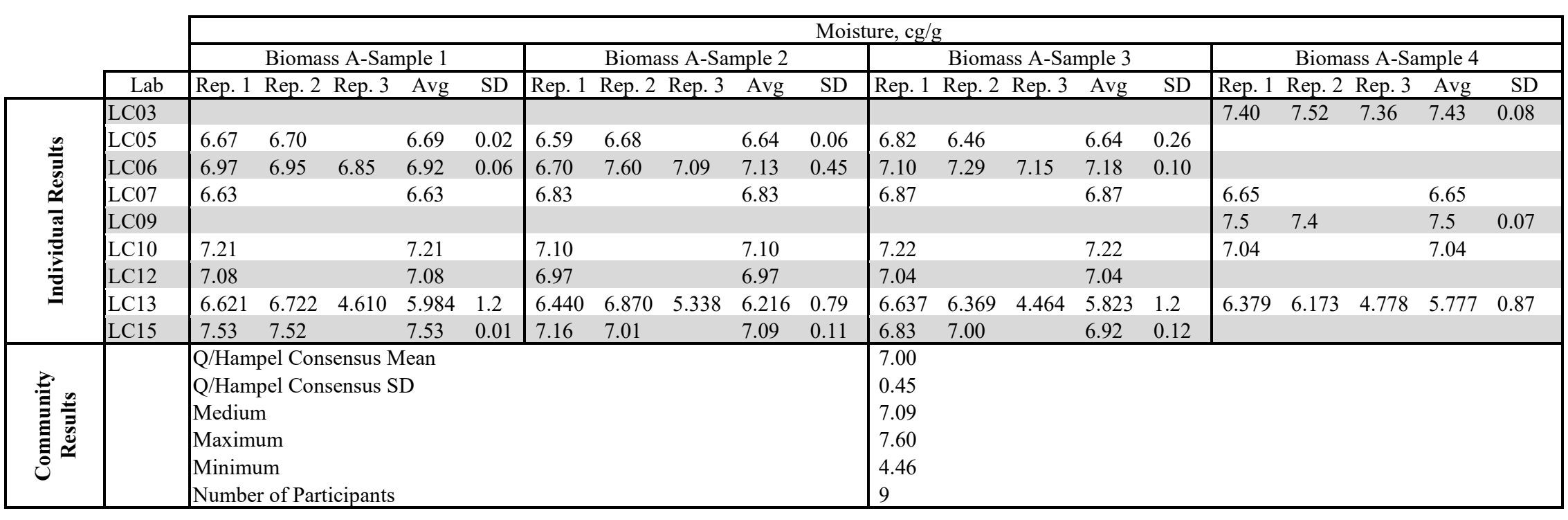




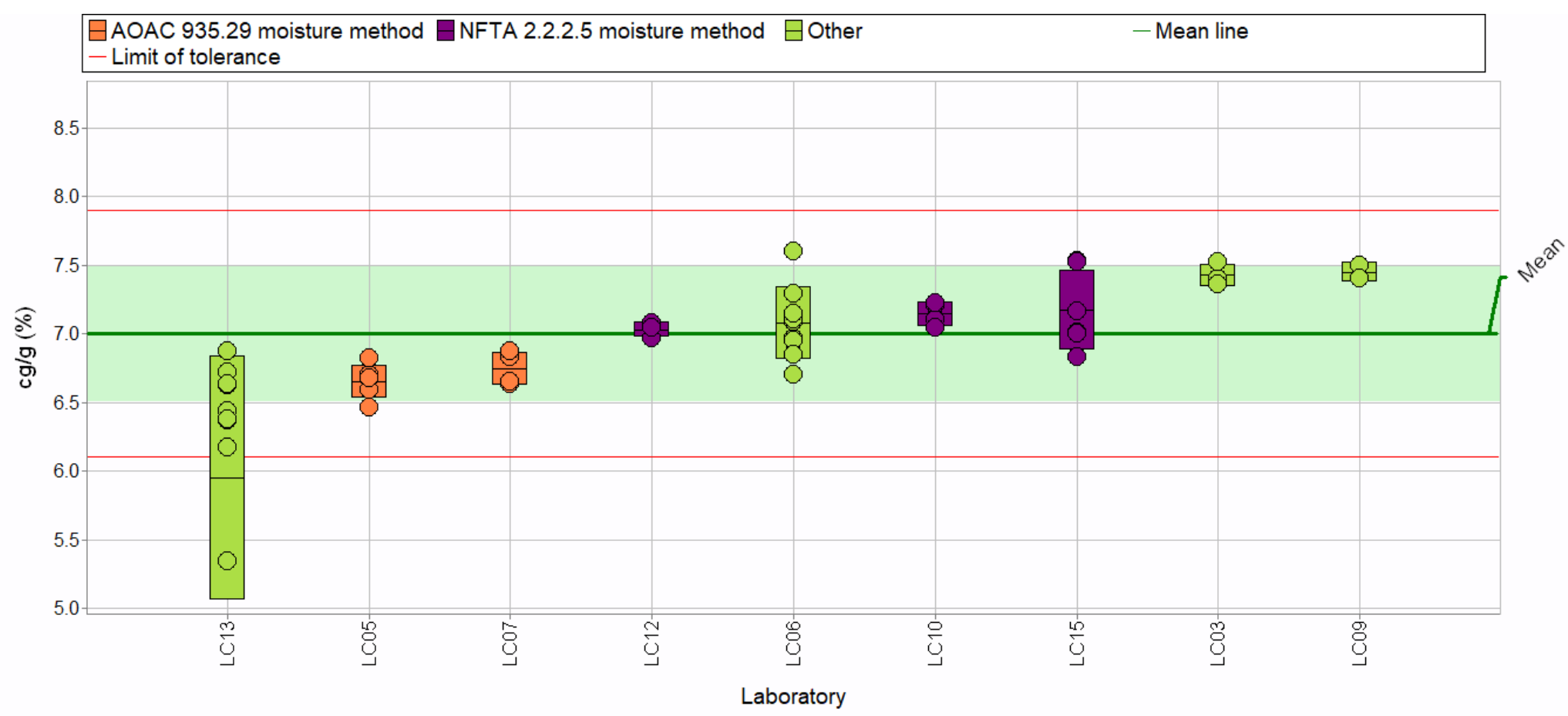

Figure 11: Mass fraction of moisture content in Biomass A.

In this view, individual laboratory data are plotted (circles) with individual laboratory SD (rectangle) for all replicates in Biomass ASample 1, Sample 2, Sample 3, and Sample 4. The color of the data points represents the method employed by each individual laboratory (see legend). The solid green line represents the consensus mean, and the light green shaded region represents the $95 \%$ confidence interval of the consensus mean. The red solid lines represent the consensus range of tolerance, calculated as the values above and below the consensus mean that result in $|\mathrm{Z}| \leq 2$. 
Table 10: Data summary for mass fraction of moisture in Biomass B.

Individual laboratory results, as reported for Samples 1-4, are listed. The community results, including the consensus mean and SD of the interlaboratory study determined by the Q/Hampel method, are listed along with the median, maximum, and minimum values reported and the number of participants that reported results.

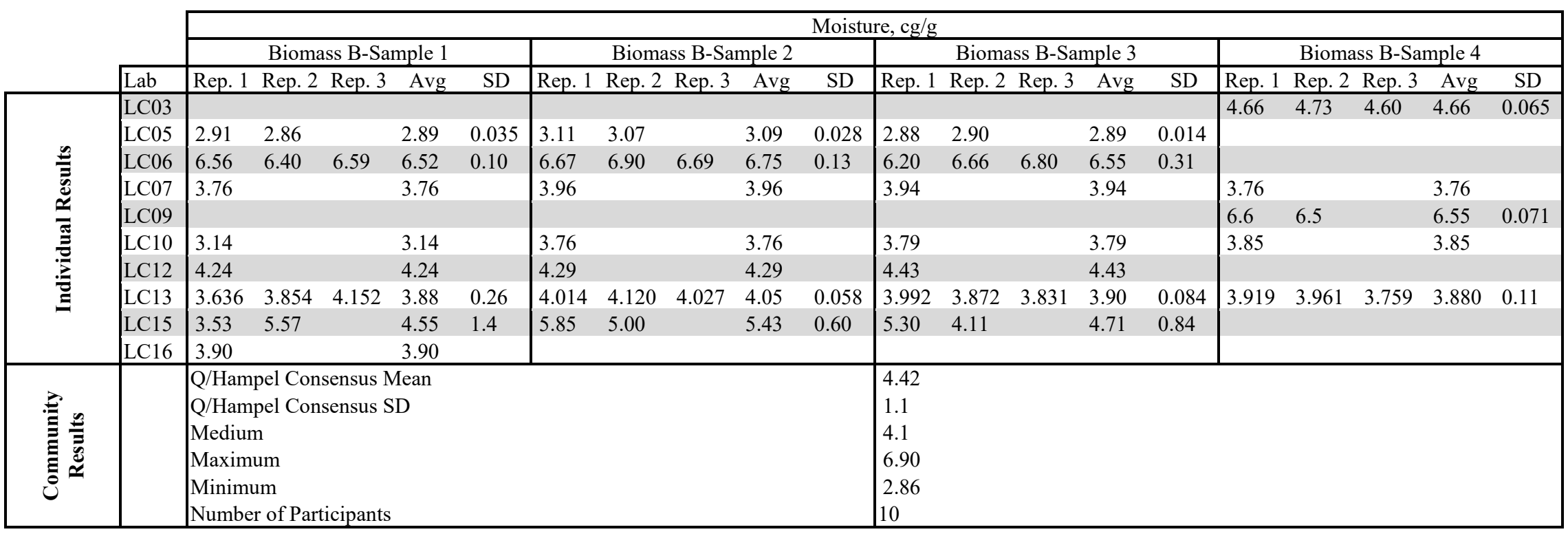




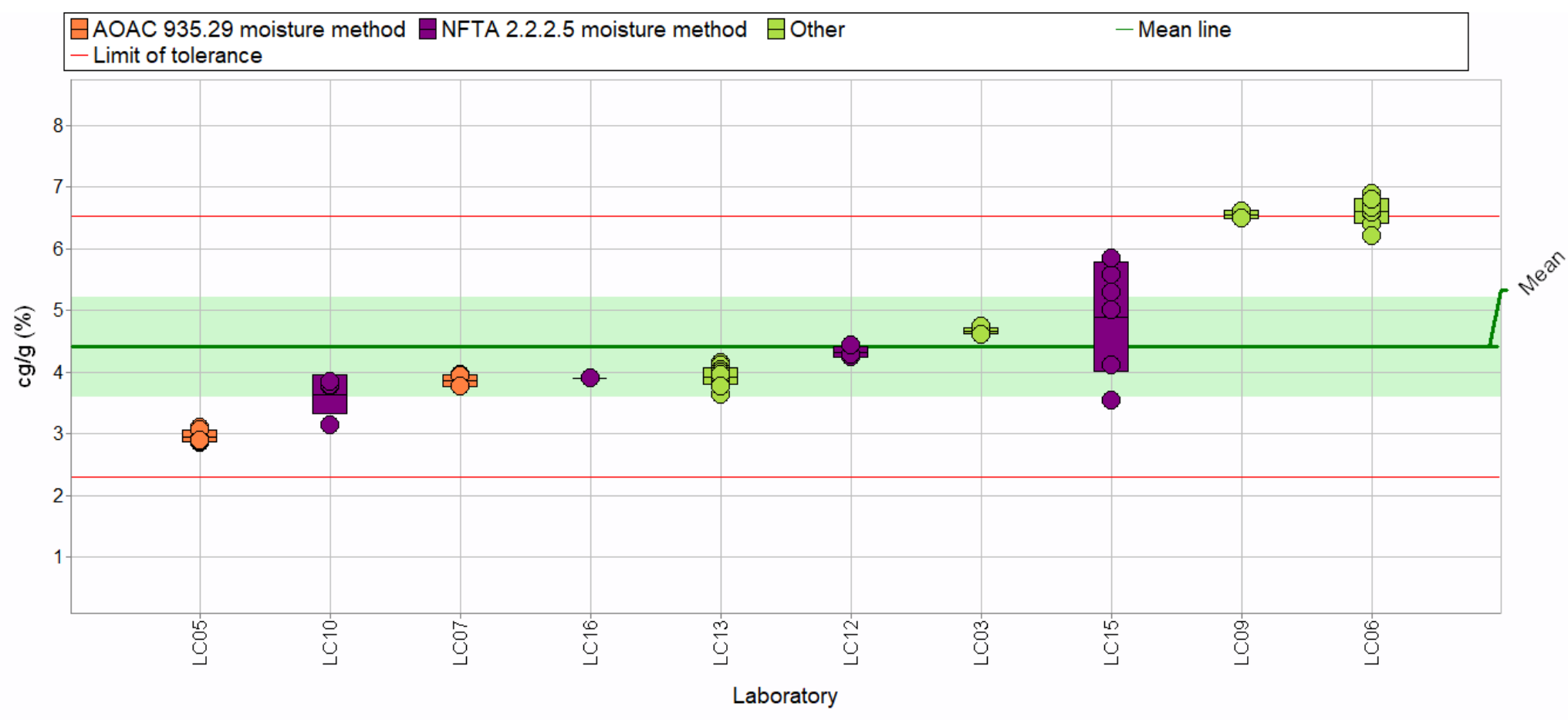

Figure 12: Mass fraction of moisture content in Biomass B.In this view, individual laboratory data are plotted (circles) with individual laboratory SD (rectangle) for all replicates in Biomass B-Sample 1, Sample 2, Sample 3, and Sample 4. The color of the data points represents the method employed by each individual laboratory (see legend). The solid green line represents the consensus mean, and the light green shaded region represents the $95 \%$ confidence interval of the consensus mean. The red solid lines represent the consensus range of tolerance, calculated as the values above and below the consensus mean that result in $|Z| \leq 2$. 
To determine if moisture correction influences the variability of the results of the interlaboratory study, the results reported both as-is and on a dry-mass basis were compared for participants that reported both starch and moisture values. For the comparison, a laboratory percent starch as-is value was converted using its own reported value for moisture. For example, the starch as-is value for Lab 5 was converted to a dry-mass basis using the average moisture content reported by Lab 5. Only ten out of the fifteen participants values could be compared since five participants did not report moisture content.

Table 11 compares the RSD of the starch values reported as-is to the RSD of starch values reported on a dry-mass basis. Reporting values on a dry-mass basis does not significantly increase the variability.

Table 11: Consensus mean, SD, and RSD of starch reported as-is and on a dry-mass basis ${ }^{\mathrm{a}}$

\begin{tabular}{|l|ccc|ccc|}
\cline { 2 - 7 } \multicolumn{1}{c|}{} & \multicolumn{6}{c|}{ Starch, cg/g } \\
\cline { 2 - 7 } \multicolumn{1}{c|}{} & \multicolumn{3}{c|}{ As-Is Basis } & \multicolumn{3}{c|}{ Dry-Mass Basis } \\
\cline { 2 - 7 } \multicolumn{1}{c|}{} & Mean $^{\mathrm{b}}$ & SD $^{\mathrm{b}}$ & RSD, \% & Mean $^{\mathrm{b}}$ & SD $^{\mathrm{b}}$ & RSD, \% \\
\hline Control A & 64.5 & 3.4 & 5.3 & 70.6 & 3.9 & 5.5 \\
Control B & 9.1 & 1.3 & 14 & 9.6 & 1.4 & 15 \\
Biomass A & 65.9 & 3.7 & 5.6 & 70.9 & 4.2 & 5.9 \\
Biomass B & 3.7 & 1.5 & 41 & 3.9 & 1.6 & 41 \\
\hline
\end{tabular}

a The consensus mean and SD listed here are based on data from participants that reported both starch and moisture content (10 participants versus 15 participants that reported starch content).

$\mathrm{b}$ The percent starch on a dry-mass basis was calculated for each starch measurement reported by participants that reported both starch and moisture content using the average moisture content for each of the individual participants (for example, the average moisture content reported by lab 5 was used to convert all of lab 5 percent starch as-is to a percent starch, dry-mass basis).

\section{Cellulose Content:}

Five participants reported data for either cellulose (to include only C6 glucan) or cellulosic content (to include cellulose + hemicellulose + lignin). Four participants reported cellulose values and two participants reported cellulosic content. One of the participants reported values for both cellulose and cellulosic content. Because of the small sample set, the results are not included in the report. It is anticipated that more participants will return data for cellulose in future studies. 


\section{Conclusions}

This report summarizes the results from an interlaboratory study for the determination of starch in corn grain biomass intermediates. Four separate materials were distributed to fifteen participating laboratories. Two of the materials were control materials gravimetrically prepared by adding known amounts of starch and cellulose (Control A and Control B). Control A consisted of both soluble and resistant starch, while Control B only consisted of soluble starch. The other two materials are candidate RMs consisting of dried corn biomass intermediates that were obtained from commercial ethanol production facilities; one was obtained before conversion (Biomass A) and the other material was obtained after conversion (Biomass B). The goals of the interlaboratory study were to evaluate the corn-to-ethanol industry's starch measurement comparability and to evaluate the two candidate RMs as being fit-for-purpose quality control materials.

The consensus value of the interlaboratory study for Control A was $4.4 \%$ lower compared to the gravimetric value. Some participants provided results that were biased low for this sample, most likely due to the inability to access the resistant starch in the sample. The ability to access the resistant starch is important when determining the starch and cellulosic converted fraction of corn kernel fiber. Ten out of the fifteen participants reported using a pretreatment procedure (such as $\mathrm{NaOH}, \mathrm{KOH}$, or DMSO) prior to enzymatic assay in order to access the resistant starch. However, results provided by some of these participants were still biased low. It is recommended that analytical methods be verified by analyzing RMs or in-house quality assurance samples that contain resistant starch. The consensus mean for starch in Control B was only slightly lower $(0.7$ $\%$ different) compared to the gravimetric value. This control material did not contain resistant starch.

The consensus mean for starch in Biomass A was determined to be $65.6 \mathrm{cg} / \mathrm{g}$ with a consensus SD of $3.8 \mathrm{cg} / \mathrm{g}$. The consensus mean for starch in Biomass B was determined to be $3.5 \mathrm{cg} / \mathrm{g}$ with a consensus SD of $1.5 \mathrm{cg} / \mathrm{g}$. The variability among the different participants is expected to improve with additional interlaboratory studies and availability of RMs. Measurement comparability is also likely to improve with open discussion of currently used analytical methods.

This interlaboratory study did not investigate the materials and methods used for calibration. It is recommended that participants use glucose with established purity or certified RMs for calibration when possible, to ensure source materials for calibration are of the highest purity.

The intralaboratory precision for samples of the same material was good for most participants as indicated by low RSDs among replicates and samples of the same material. Ensuring that the samples are mixed thoroughly before aliquoting for analysis and the use of larger sampling sizes are a few suggestions for improving precision.

The within- and between-sample variations for Biomass A and Biomass B determined by participants that reported replicates for multiple samples of the same material were low. This suggests that the materials are homogenous for the sampling sizes required by the methods commonly used in the industry. 
Participants were asked to report starch on an as-is basis and thus not correct for moisture content. However, participants were given the option of reporting moisture content. There was generally good agreement among participants reporting moisture. It was determined that correcting for moisture did not increase the variability of the starch values. However, for comparability of future results if reporting on a dry-mass basis, it is recommended that participants use a common moisture method.

NIST intends to further develop Biomass A and Biomass B as RMs. The data reported here (along with additional data obtained at NIST) may be used for the value assignment of starch in these two materials. The availability of a corn biomass-based RM that is like samples that will be routinely analyzed in the corn-to-ethanol industry will help improve comparability among participants and to evaluate and improve methods. Future work also includes examining cellulose in the materials by conducting follow-on interlaboratory studies with these materials. 


\section{Acknowledgements}

I thank my NIST colleagues David Duewer, Katrice Lippa, and Hung-Kung Liu for their insightful input in statistical analysis of interlaboratory data and for their editorial assistance. I thank my NIST colleagues Jennifer Benkstein, Mark Cronise, Curtis Fales, and Thomas Shuggars for their help with material documentation and packaging the candidate RMs. I thank Brandon Emme of ICM Research and Development, Kurt Gustafson of the U.S. Environmental Protection Agency, Kristi Plack of Soliton, Justin Sluiter of the National Renewable Energy Laboratory, Shon Van Hulzen of POET, and Yanhong Zhang of the National Corn-to-Ethanol Research Center for their suggestions and guidance in developing the candidate RMs. The time and effort of the analysts and management of the participating laboratories are gratefully acknowledged.

\section{References}

1. U.S. Environmental Protection Agency. Renewable Fuel Standard Program Home Page. https://www.epa.gov/renewable-fuel-standard-program (accessed Dec 30, 2019).

2. U.S. Environmental Protection Agency. Regulation of Fuels and Feul Additives: RFS Pathways II, and Technical Amendments to the RFS Standards and E15 Misfueling Mitigation Requariqirments. Fed Regist. 2014, 79, 42127-42167.

3. How are RINs Generated and Assigned to Batches of Renewable Fuel by Renwable Fuel Producers or Importers? Table 1; Code of Federal Regulations, Section 80.1426, subpart M. Title 40.

4. Guidance on Qualifying an Analytical Method for Determining the Cellulosic Converted Fraction of Corn Kernel Fiber Co-Processed with Starch, EPA-420-B-19-022, Compliance Division Office of Transporation and Air Quality, U. S. Environmental Proetection Agency:

https://www.epa.gov/renewable-fuel-standard-program/guidance-qualifying-analytical-methoddetermining-cellulosic. May 2019.

5. Official Method of Analysis of AOAC International (2005) $18^{\text {th }}$ Ed., AOAC International, Gaithersburg, MD, USA, Official Method 996.11 Starch (Total) in Cereal Products.

6. Official Method of Analysis of AOAC International (2005) $18^{\text {th }}$ Ed., AOAC International, Gaithersburg, MD, USA, Official Meethod 935.29 Moisture in Malt.

7. National Forage Testing Association Reference Method: Dry Matter by Oven Drying for 3 Hours at $105^{\circ} \mathrm{C}$. National Forage Testing Association: Omaha, NE, USA, 2006.

8. Clinical and Laboratory Standards Institute (CLS). Expression of Measurment Uncertainty in Laboratory Medicine; Approved Guideline. CLSI document C51-A. Wayne, Pennsylavania 19087 USA, 2012.

9. International Organization for Standardization (2015), Geneva, Switzerland. Statistical methods for use in proficiency testing by interlaboratory comparison (ISO 13528:2015). 